\title{
Spatial development of a turbulent boundary layer subjected to freestream turbulence
}

\author{
Yannick Jooss ${ }^{1}$, Leon $\mathrm{Li}^{1}$, Tania Bracchi ${ }^{1}$ and R. Jason Hearst ${ }^{1}{ }^{\dagger}$ \\ ${ }^{1}$ Department of Energy and Process Engineering, Norwegian University of Science and Technology, \\ Trondheim NO-7491, Norway
}

(Received 5 June 2020; revised 18 September 2020; accepted 28 October 2020)

The spatial development of a turbulent boundary layer (TBL) subjected to freestream turbulence (FST) is investigated experimentally in a water channel for friction Reynolds numbers up to $R e_{\tau}=5060$. Four different FST intensities are generated with an active grid, ranging from a low-turbulence reference case to $u_{\infty}^{\prime} / U_{\infty}=12.5 \%$. Wall-normal velocity scans are performed with laser doppler velocimetry at three positions downstream of the grid. There are two combating influences as the flow develops: the TBL grows while the FST decays. Whilst previous studies have shown the wake region of the TBL is suppressed by FST, the present measurements demonstrate that the wake recovers sufficiently far downstream. For low levels of FST, the near-wall variance peak grows as one moves downstream, whereas high FST results in an initially high variance peak that decays with streamwise position. These results are mirrored in the evolution of the spectrograms, where low FST results in the emergence of an outer spectral peak as the flow evolves, while high FST sees an initially high outer spectral peak decay in space. This finding is significant as it suggests the FST does not permanently mature the TBL ahead of its natural evolution. Finally, it is explicitly demonstrated that it is not sufficient to characterize the TBL solely by conventional parameters such as $R e_{\tau}$, but that the level of FST and the evolution of the two flows must also be considered.

Key words: homogeneous turbulence, turbulent boundary layers

\section{Introduction}

Turbulent boundary layers (TBL) exist in a wide range of natural processes and technical applications. Understanding their nature and evolution has been a subject of great interest since the concept was first introduced (Prandtl 1905). The study of TBLs is also important for developing knowledge on diverse problems ranging from how heat is distributed in the atmosphere to the determination of drag forces on aeroplanes and ships (Smits \& Marusic 2013). In many of these flows, the freestream above the boundary layer is also turbulent. The characteristics of the so-called freestream turbulence (FST) can vary significantly; two parameters of leading-order significance are the turbulence intensity $u_{\infty}^{\prime} / U_{\infty}$, where $U_{\infty}$ is the freestream velocity and $u_{\infty}^{\prime}$ is the root-mean-square of the

$†$ Email address for correspondence: jason.hearst@ntnu.no 
velocity fluctuations in the freestream, and the size of the largest scales in the flow, both of which vary depending on the turbulence's origin and state of evolution. Over the past three decades the effect of FST on a canonical zero-pressure-gradient turbulent boundary layer has been studied extensively, e.g. Hancock \& Bradshaw (1983, 1989), Castro (1984), Thole \& Bogard (1996), Sharp, Neuscamman \& Warhaft (2009), Dogan, Hanson \& Ganapathisubramani (2016), Dogan, Hearst \& Ganapathisubramani (2017), Hearst, Dogan \& Ganapathisubramani (2018), Dogan et al. (2019) and You \& Zaki (2019).

Pioneering work in subjecting a turbulent boundary layer to FST was performed by Hancock \& Bradshaw $(1983,1989)$. Freestream turbulence was generated with two different passive grids in a wind tunnel, and the flow was measured over a flat plate. The freestream turbulence intensity and length scales were also varied by measuring at different downstream positions from the grids. This resulted in a range of $2870 \leqslant$ $\operatorname{Re}_{\theta} \leqslant 5760$, where $\operatorname{Re}_{\theta}=U_{\infty} \theta / v$ is based on the momentum thickness $\theta$. They covered a range of freestream turbulence length scales $L_{u, \infty}$, representing the characteristic length scale of the energy containing eddies, between 0.67 and 2.23 times the boundary layer thickness $\delta$. They found both $u_{\infty}^{\prime} / U_{\infty}$ and $L_{u, \infty}$ were significant influencing parameters on the structure of the boundary layer. They combined these concepts in an empirical parameter, $\beta=\left(u_{\infty}^{\prime} / U_{\infty}\right) /\left(L_{u, \infty} / \delta+2\right)$, which appeared to correlate well with the wall shear stress and boundary layer wake region in their flows. However, their experiment was not without limitations - for example, the relatively low turbulence intensities, up to a maximum of $5.8 \%$, and, more importantly, measurement positions as close as 15 mesh lengths $(M)$ downstream of their grids where the flow is typically still inhomogeneous (Ertunç et al. 2010; Isaza, Salazar \& Warhaft 2014). The measurement position relative to the grid bars could bias the results in this region, and more recent measurements offer words of caution and update these results (Hearst et al. 2018; Kozul et al. 2020). Several other fluids problems, including flow over aerofoils, for example, have shown sensitivity to being in the inhomogeneous region behind a grid, resulting in strongly contrasting results (Devinant, Laverne \& Hureau 2002; Wang et al. 2014; Maldonado et al. 2015). Castro (1984) looked at the effect of freestream turbulence on turbulent boundary layers at relatively low Reynolds numbers, $500 \leqslant R e_{\theta} \leqslant 2500$. Two passive grids were used to create the FST with turbulence intensities up to $7 \%$. It was shown that the skin friction was influenced by both the Reynolds number and the freestream turbulence intensity. Once again measurements were, in part, taken relatively close to the grid, starting from $x / M=6$.

Similarly, Blair (1983b) showed that the skin friction increases with FST in a turbulent boundary layer for $1000 \leqslant R e_{\theta} \leqslant 7000$. In the second part of his work (Blair 1983a), the influence of FST on the shape of the turbulent boundary layer profile was analysed. While the logarithmic region was relatively unaffected by the freestream turbulence, the presence of the wake was found to be strongly dependent on the level of FST. The outer region intermittency was progressively suppressed with increasing turbulence intensity, effectively making the wake region of the boundary layer profile imperceptible for $u_{\infty}^{\prime} / U_{\infty} \gtrsim 5.3 \%$.

A different way to introduce FST was examined by Thole \& Bogard (1996). Crossflow jets were used to generate turbulence intensities up to $20 \%$ in the freestream. The conclusions remained the same with the wake being suppressed while the logarithmic region was maintained. This demonstrated that it is not pivotal how the FST is generated.

In a study of canonical turbulent boundary layers without FST, Hutchins \& Marusic (2007) introduced the use of spectrograms in boundary layer research. Pre-multiplied spectra at different wall-normal positions throughout the boundary layer are plotted in a contour map illustrating the energy distribution between different wavelengths in the 
boundary layer from the wall up to the freestream. They covered a range of friction Reynolds numbers $1010 \leqslant R e_{\tau} \leqslant 7300$, with $R e_{\tau}=U_{\tau} \delta / v$ based on the friction velocity $U_{\tau}$. Two peaks were found in the spectrograms: one coinciding with the location of the variance peak close to the wall, which was present through the full range of $R e_{\tau}$ examined, and an outer peak emerging with increasing $R e_{\tau}$, distinctly visible at $R e_{\tau}=7300$. Sharp et al. (2009) were the first to use an active grid to study the influence of FST on turbulent boundary layers. The active grid was modeled after the original design of Makita (1991). With the active grid, FST intensities up to $10.5 \%$ were produced. This corresponded to a turbulence Reynolds number of $R e_{\lambda}=550$, with $R e_{\lambda}=u_{\infty}^{\prime} \lambda_{\infty} / v$ based on the Taylor microscale $\lambda_{\infty}$. The examined boundary layers $\left(550 \leqslant R e_{\theta} \leqslant 2840\right)$ showed a decrease of the wake strength with increasing FST, consistent with Blair (1983a). Analysing the pre-multiplied energy spectra showed the emergence of an outer spectral peak similar to the findings of Hutchins \& Marusic (2007) at considerably lower $R e_{\tau}$. This result was confirmed by Dogan et al. (2016) who also showed that the magnitude of the outer spectral peak scales with FST. In that work, turbulence intensities up to $13 \%$ were generated with an active grid, and it was shown that the streamwise velocity fluctuations at the near-wall peak in the boundary layer correlate with freestream turbulence intensity. These observations in combination with the presented energy spectra demonstrate that the FST penetrates the boundary layer down to the wall. Despite the permeance of the FST, Dogan et al. (2017) used the same setup to demonstrate that the near-wall region is statistically similar to a canonical high- $R e_{\tau}$ turbulent boundary layer without FST.

Using the same setup, Esteban et al. (2017) confirmed the increase of skin friction with growing FST (Blair 1983a; Castro 1984). Oil-film interferometry was used to obtain the wall shear stress. It was also found that the relation between Reynolds number and skin friction is similar to canonical turbulent boundary layers without FST. Furthermore, it was demonstrated that oil-film interferometry and the multi-point composite fitting technique of Rodríguez-López, Bruce \& Buxton (2015) were in good agreement in their estimates of $U_{\tau}$ for these TBL flows with FST above them.

In a subsequent study by Hearst $e t$ al. (2018), it was shown that for $8.2 \% \leqslant u_{\infty}^{\prime} / U_{\infty} \leqslant$ $12.3 \%$, corresponding to $455 \leqslant R e_{\lambda} \leqslant 615$ and up to $65 \%$ changes in the integral scale for a fixed $u_{\infty}^{\prime} / U_{\infty}$, there was no influence of the length scale on the features of the boundary layer. It was proposed that this result differed from the older Hancock \& Bradshaw (1989) result because of the increase in turbulence intensity, a different way of measuring the integral scale and measurements performed at positions more suitably distant from the grid. Through spectral analysis it was found that only the large scales penetrate the boundary layer, resulting in the outer spectral peak which would otherwise not be present in these flows, while the inner spectral peak remained unaffected. This result was included in the formulation of the law of the wall for such flows by Ganapathisubramani (2018). Finally, Hearst et al. (2018) developed a model that reproduced the spectrogram of the boundary layer based on the pre-multiplied energy spectrum of the freestream.

The majority of the aforementioned studies focussed on statistics and spectra at singular points in the TBL and did not investigate the streamwise development of the boundary layer. Earlier studies were in fact almost exclusively single plane measurements, and if the streamwise position was varied, this typically involved moving closer to the grid to obtain higher turbulence intensities. The spatial evolution of a canonical turbulent boundary layer without FST was studied experimentally by Vincenti et al. (2013) and Marusic et al. (2015). They showed that the magnitude of the near wall variance peak increases as the boundary layer evolves spatially. Furthermore, it was demonstrated that the emergence of an outer spectral peak with increasing $R e_{\tau}$ can also be observed in a spatially evolving turbulent boundary layer. There has also been some effort to simulate spatially developing 
canonical turbulent boundary layers (Ferrante \& Elghobashi 2004; Wu \& Moin 2009; Eitel-Amor, Örlü \& Schlatter 2014; Wu et al. 2017).

None of the aforementioned works investigated how a turbulent boundary layer evolves when subjected to FST which itself is also evolving. Raushan, Singh \& Debnath (2018) examined a flow of this type, posing the inverse question: how does the spatial development of a boundary layer influence grid generated freestream turbulence. They used three different passive grids in an open water channel to create different levels of freestream turbulence. The focus in their analysis was on the development of inhomogeneous turbulence in the near-field region of the grids. You \& Zaki (2019) compared a turbulent boundary layer subjected to FST (inflow $u_{\infty}^{\prime} / U_{\infty}=10 \%$ ) to a canonical TBL in a direct numerical simulation (DNS). At $1900 \leqslant R e_{\theta} \leqslant 3000$, an increase of the skin-friction of up to $15 \%$ was observed in the presence of FST, as well as the suppression of the wake region, confirming previous experimental results. This study also affirmed an increase in magnitude of the near-wall streamwise variance peak with the logarithmic region remaining robust. At their highest $R e_{\theta}=3000$, they also observed the emergence of an outer peak in the pre-multiplied energy spectrogram. Wu, Wallace \& Hickey (2019) examined the interfaces between freestream turbulence and laminar and turbulent boundary layers, as well as turbulent spots in a DNS, for $80 \leq R e_{\theta} \leq 3000$. Recently, Kozul et al. (2020) explored the evolution of a temporal turbulent boundary layer subjected to decaying FST. In their DNS study, they analysed the relative timescales of boundary layers and freestream turbulence to determine if and how much the boundary layer is affected. These were insightful works, but the achievable Reynolds numbers in DNS studies are still relatively low compared to what can be realized in a laboratory. So far the development of a turbulent boundary layer subjected to freestream turbulence has only been studied for low Reynolds numbers $\left(R e_{\tau}, R e_{\theta}\right)$ and in single cases without comparison to other FST parameters. This study addresses this gap by examining the development of a turbulent boundary layer for $R e_{\tau}>5000$ and $R e_{\theta}>9000$ at three states of evolution for four levels of freestream turbulence. The influence of the evolving freestream turbulence on the mean velocity and variance profiles is examined, as well as the spectral distribution of energy in the developing boundary layer.

\section{Experimental methods and procedure}

The measurements were conducted in the water channel at the Norwegian University of Science and Technology. A schematic of the facility is provided in figure 1 . The test section measures $11 \mathrm{~m} \times 1.8 \mathrm{~m} \times 1 \mathrm{~m}$ (length $\times$ width $\times$ height) with a maximum water depth of $0.8 \mathrm{~m}$. It is a recirculating, free surface, water channel with a $4: 1$ contraction followed by an active grid upstream of the test section. A $10 \mathrm{~mm}$ thick acrylic plate measuring $1.8 \mathrm{~m} \times 1.045 \mathrm{~m}$ was placed at the start of the test section, immediately downstream of the active grid, on the water surface to dampen surface waves directly caused by the water flowing through the bars of the active grid; the remaining $\sim 10 \mathrm{~m}$ of the water channel has a free surface. More details on the facility can be found in appendix A.

The active grid used in this study to generate the freestream turbulence is based on the design of Makita (1991). It is a biplanar grid with 28 rods - 10 horizontal and 18 vertical (figure 2). The rods are equipped with square-shaped wings that measure $100 \mathrm{~mm}$ on the diagonal and include two holes to reduce the motor loading, as well as to prevent $100 \%$ blockage from occurring. Each rod can be controlled independently with a stepper motor. The mesh length of the grid, i.e. the spacing between each rod, is $M=100 \mathrm{~mm}$. More information on the active grid design is provided in appendix B. 


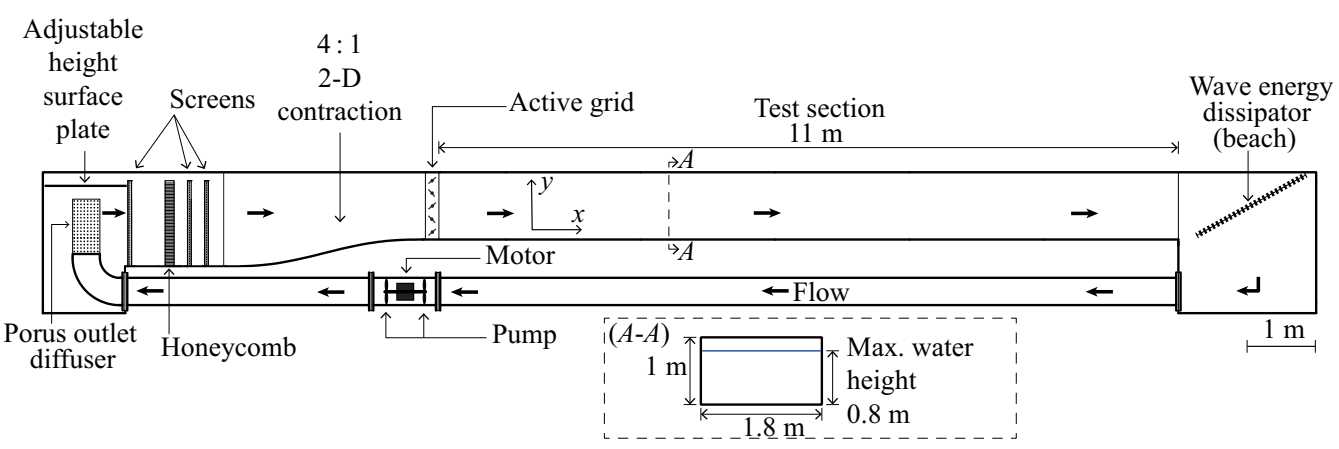

FIGURE 1. Schematic of the water channel facility in Strømningslaben at the Norwegian University of Science and Technology.

(a)

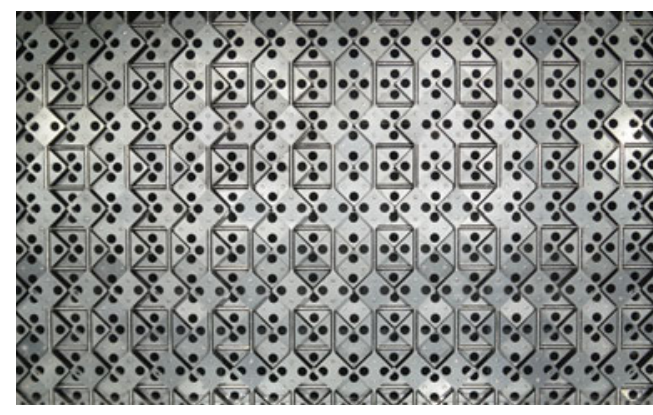

(b)

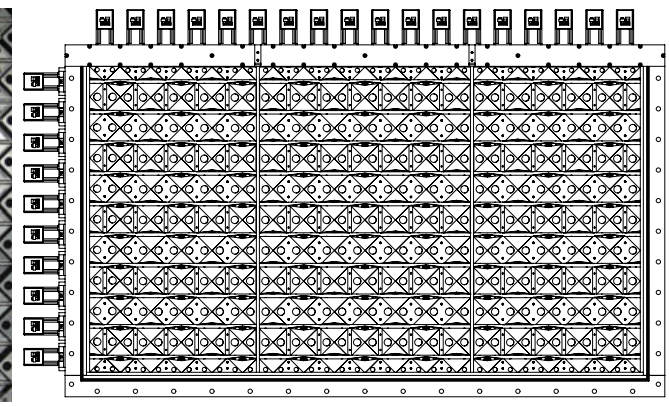

FIGURE 2. Biplanar active grid featuring square wings with holes. Viewed from the test section at full blockage and full schematic of the active grid.

The boundary layer was tripped by the bars of the active grid and then allowed to develop along the glass floor of the water channel. Wall-normal boundary layer scans were performed in the centre of the channel at three streamwise positions, $x / M=35,55$, and 95. The downstream positions relative to the grid were chosen to be greater than $30 \mathrm{M}$ to be in keeping with grid turbulence norms for homogeneity and isotropy of the freestream at all measurement positions (Ertunç et al. 2010; Isaza et al. 2014; Hearst \& Lavoie 2015). Velocity measurements were performed with single-component laser doppler velocimetry (LDV). The laser has a wavelength of $514.5 \mu \mathrm{m}$. A $60 \mathrm{~mm}$ FiberFlow probe from Dantec Dynamics was used in backscatter mode in combination with a beam expander and a lens with a focal length of $500 \mathrm{~mm}$. This results in an elliptical measuring volume with dimensions $\mathrm{d} x \times \mathrm{d} y \times \mathrm{d} z=119 \mu \mathrm{m} \times 119 \mu \mathrm{m} \times 1590 \mu \mathrm{m}$, which corresponds to 1.6-1.8 wall units $y^{+}$in the wall-normal direction (depending on the case) and a fringe spacing of $3.33 \mu \mathrm{m}$. Wall unit normalization of the wall-normal position is $y^{+}=y U_{\tau} / \nu$. The wall was found by manually positioning the measurement volume near the wall and then traversing downward in $0.1 \mathrm{~mm}$ steps until the data rate suddenly increased, indicating reflections by the glass floor. This gives an accuracy of $\sim 0.05 \mathrm{~mm}$. The probe was then traversed upward from this position to the water surface applying a logarithmic spacing with a total of 24 measurement points for each scan. A method to correct for the true wall-normal position from the mean velocity profile, introduced by Rodríguez-López et al. (2015), was applied $a$ posteriori. 
The sampling rate of LDV is non-constant and varies with mean velocity - thus, in this study effectively with wall-normal distance. The mean sampling rate varied between $7 \mathrm{~Hz}$ directly at the wall and $155 \mathrm{~Hz}$ in the freestream. To guarantee convergence throughout the scans, every position was sampled for $10 \mathrm{~min}$. This is between 630 and 1440 boundary layer turn-overs for a single measurement, depending on the test case. This might be low compared to some hot-wire studies, but it is still a substantial amount of data and sampling time with a single scan, pushing the realistic limits for what could be accomplished as a continuous run. Moreover, a 20 min convergence study in the freestream for the most turbulent case showed only a $0.4 \%$ change in the variance compared to 10 min samples, which is smaller than the other measurement uncertainties. Time-series acquired with LDV also have a non-uniform time step distribution. To perform spectral analysis it is therefore required to resample the data. This is done with sample and hold reconstruction as proposed by Boyer \& Searby (1986) and Adrian \& Yao (1986). This method returns a uniformly spaced data series, which can then be used to compute spectra using a fast Fourier transform in the same manner as hot-wire data. The spectra are filtered with a bandwidth moving filter of $25 \%$ to facilitate the identification of the underlying trends (Baars, Hutchins \& Marusic 2016).

The friction velocity, $U_{\tau}$, was estimated from the measured velocity profiles using the method introduced by Rodríguez-López et al. (2015), which was demonstrated to be effective in these flows by comparison to oil-film interferometry (Esteban et al. 2017). This method is essentially a multi-variable optimization applied to the composite boundary layer profile,

$$
U^{+}=\frac{1}{\kappa} \ln \left(y^{+}\right)+C^{+}+\frac{2 \Pi}{\kappa} \mathcal{W}\left(\frac{y^{+}}{R e_{\tau}}\right),
$$

where $\kappa$ is the von Kármán constant, $\Pi$ is Coles' wake parameter (Coles 1956) and $\mathcal{W}$ is the wake function defined as per Chauhan, Monkewitz \& Nagib (2009). Due to a limited number of points acquired in the log-region, a simple comparison of $\kappa$ to $\kappa=0.39 \pm 0.02$ as found by Marusic et al. (2013) across several facilities was made and found to be in good agreement; this is illustrated explicitly in the subsequent figures. The von Kármán constant is not a specific focus of the present investigation, but the interested reader can find more details on $\kappa$ in the work by Hearst et al. (2018), who measured several points within the log-region for a TBL subjected to FST.

\section{Freestream conditions}

Four different inflow conditions were investigated in this work. They are presented in table 1 with their freestream statistics at the three measurement positions. The mean velocity in the freestream was kept constant at $U_{\infty}=0.345 \pm 0.015 \mathrm{~m} \mathrm{~s}^{-1}$ for all test cases. A slight increase in velocity was recorded for the downstream positions. This is expected due to the head loss and growing boundary layer in an open channel flow. Overall the differences in mean velocity are considered negligible here. The parameter of interest that was deliberately varied between cases is the turbulence intensity in the freestream $u_{\infty}^{\prime} / U_{\infty}$. The reference case (REF) was created by orienting all the wings of the active grid in line with the flow, resulting in $2.5 \% \leq u_{\infty}^{\prime} / U_{\infty} \leq 3.2 \%$ at the three measurement positions. It is worth noting that the background turbulence in water channel flows is typically on the order of 2 or $3 \%$, and thus this particular case quickly sees the flow return to the background state of the water channel. For comparison, the canonical turbulent boundary layer results presented by Laskari et al. (2018) were measured in a water channel with $\sim 3 \%$ turbulence intensity in the freestream; thus our REF case is 


\begin{tabular}{|c|c|c|c|c|c|c|c|c|}
\hline Case & $\begin{array}{c}\Omega \pm \omega \\
(\mathrm{Hz})\end{array}$ & $x / M$ & $\begin{array}{c}U_{\infty} \\
\left(\mathrm{m} \mathrm{s}^{-1}\right)\end{array}$ & $\underset{(\%)}{u_{\infty}^{\prime} / U_{\infty}}$ & $R e_{\lambda, \infty}$ & $\begin{array}{l}L_{u, \infty} \\
(\mathrm{m})\end{array}$ & $u_{\infty}^{\prime} / v_{\infty}^{\prime}$ & Symbol \\
\hline REF & - & $\begin{array}{l}35 \\
55 \\
95\end{array}$ & $\begin{array}{l}0.33 \\
0.34 \\
0.35\end{array}$ & $\begin{array}{l}3.2 \\
2.9 \\
2.5\end{array}$ & $\begin{array}{l}59 \\
52 \\
45\end{array}$ & $\begin{array}{l}0.20 \\
0.24 \\
0.32\end{array}$ & $\begin{array}{l}1.1 \\
1.2 \\
1.2\end{array}$ & 0 \\
\hline A & $\begin{array}{c}1 \pm 0.5 \\
(2 \mathrm{D})\end{array}$ & $\begin{array}{l}35 \\
55 \\
95\end{array}$ & $\begin{array}{l}0.34 \\
0.34 \\
0.35\end{array}$ & $\begin{array}{l}5.5 \\
4.7 \\
3.8\end{array}$ & $\begin{array}{l}176 \\
142 \\
103\end{array}$ & $\begin{array}{l}0.30 \\
0.37 \\
0.50\end{array}$ & $\begin{array}{l}1.2 \\
1.2 \\
1.2\end{array}$ & 口 \\
\hline B & $1 \pm 0.5$ & $\begin{array}{l}35 \\
55 \\
95\end{array}$ & $\begin{array}{l}0.34 \\
0.34 \\
0.35\end{array}$ & $\begin{array}{l}7.4 \\
6.0 \\
5.0\end{array}$ & $\begin{array}{l}303 \\
219 \\
176\end{array}$ & $\begin{array}{l}0.39 \\
0.49 \\
0.64\end{array}$ & $\begin{array}{l}1.2 \\
1.2 \\
1.2\end{array}$ & $\begin{array}{l}\Delta \\
\Delta\end{array}$ \\
\hline $\mathrm{C}$ & $0.1 \pm 0.05$ & $\begin{array}{l}35 \\
55 \\
95\end{array}$ & $\begin{array}{l}0.35 \\
0.35 \\
0.36\end{array}$ & $\begin{array}{r}12.5 \\
9.6 \\
7.7\end{array}$ & $\begin{array}{l}725 \\
495 \\
392\end{array}$ & $\begin{array}{l}0.50 \\
0.69 \\
0.94\end{array}$ & $\begin{array}{l}1.2 \\
1.1 \\
1.2\end{array}$ & 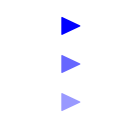 \\
\hline
\end{tabular}

TABLE 1. Freestream parameters of the examined cases at the different streamwise positions. Note that the colours fade with increasing downstream distance from the grid. These symbols are used in all figures and tables.

equivalent to their canonical case. For case A, the wings on the vertical rods remained static, while the horizontal rods were actuated. For the last two cases, B and C, all rods were actuated. The actuation mode for the cases $\mathrm{A}-\mathrm{C}$ was always fully random. This means rotational velocity, acceleration and period were varied randomly over a set range (Hearst $\&$ Lavoie 2015). The parameter that was varied between cases was the mean rotational velocity $\Omega$, i.e. $\Omega_{A},=\Omega_{B}=1 \mathrm{~Hz}$ and $\Omega_{C}=0.1 \mathrm{~Hz}$. All three cases were varied with a top-hat distribution $\Omega \pm \omega$ with the limits $\omega=0.5 \Omega$. The exact distributions used for each case are listed in table 1 . The period and acceleration were always varied in the same range of $0.5-10 \mathrm{~s}$ and $10-100 \mathrm{~s}^{-2}$, respectively. The parameters were chosen based on the findings of previous active grid studies (Kang, Chester \& Meneveau 2003; Larssen \& Devenport 2011; Hearst \& Lavoie 2015; Hearst et al. 2018) and slightly adapted to reflect the requirements of this study. The result is a wide range of turbulence intensities at the first measurement position $x / M=35$, from $3.2 \%$ for REF up to $12.5 \%$ for case $\mathrm{C}$. The turbulence intensity at the first position will be referred to as the initial turbulence intensity, $u_{0}^{\prime} / U_{0}=\left(u_{\infty}^{\prime} / U_{\infty}\right)_{x / M=35}$.

The decay of the turbulence in the freestream was measured with a finer streamwise discretization. Measurements were taken at 15 positions between $x / M=15$ and $x / M=$ 107 at $y=500 \mathrm{~mm}$. This wall-normal position was chosen as it was always outside the boundary layer while also being far away from the free surface. As the turbulence decays with increasing distance from the grid, the spread of turbulence intensity between the cases becomes smaller from $\Delta u_{\infty}^{\prime} / U_{\infty}=9.3 \%$ at $x / M=35$ down to $\Delta u_{\infty}^{\prime} / U_{\infty}=5.2 \%$ at the last measurement position, $x / M=95$. The decay of the turbulence with increasing distance from the grid can be described by a power law (Comte-Bellot \& Corrsin 1966; Mohamed \& Larue 1990; Lavoie, Djenidi \& Antonia 2007; Isaza et al. 2014),

$$
\frac{u_{\infty}^{\prime 2}}{U_{\infty}^{2}}=A\left(\frac{x}{M}-\frac{x_{0}}{M}\right)^{-n}
$$




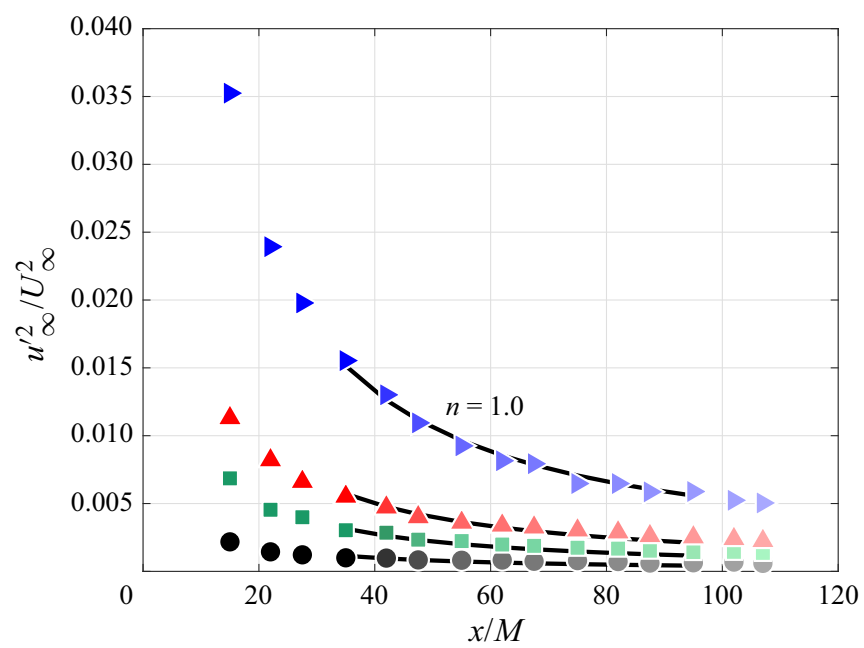

FIGURE 3. Decay of turbulence for case REF $\bullet$; A $\boldsymbol{\square}$, green; B $\boldsymbol{\Delta}$, red; C $\bullet$, blue with fading colours indicating increasing streamwise distance from the grid.

where $x_{0}$ is a virtual origin, and $A$ and $n$ are the decay coefficient and exponent, respectively. Figure 3 shows the best fits to (3.1), resulting in $n \approx 1$ for all cases. Here, all three variables, $A, x_{0}$ and $n$ were allowed to vary.

The Taylor microscale in the freestream $\lambda_{\infty}$ was calculated as

$$
\lambda_{\infty}^{2}=\frac{u^{\prime 2}}{\left\langle(\partial u / \partial x)^{2}\right\rangle},
$$

assuming local isotropy and Taylor's frozen flow hypothesis to calculate $(\partial u / \partial x)^{2}$ from the time series data acquired at a singular streamwise position. A sixth-order central differencing scheme was used to determine the gradients as suggested by Hearst et al. (2012). This leads to turbulence Reynolds numbers $R e_{\lambda}$ between 45 and 725 . A decrease of $R e_{\lambda}$ can be observed both for decreasing $u_{0}^{\prime} / U_{0}$ and with streamwise evolution of the flow, as expected.

The integral length scale $L_{u, \infty}$ was calculated as proposed by Hancock \& Bradshaw (1989) assuming isotropic turbulence,

$$
U_{\infty} \frac{\mathrm{d} u_{\infty}^{\prime}{ }^{2}}{\mathrm{~d} x}=\frac{-\left(u_{\infty}^{\prime}{ }^{2}\right)^{3 / 2}}{L_{u, \infty}}
$$

where $x$ is the downstream distance from the grid, and the gradient $\mathrm{d} u_{\infty}^{\prime}{ }^{2} / \mathrm{d} x$ is calculated in physical space by taking the analytical derivative of (3.1). An increase in $L_{u, \infty}$ exists as the distance from the grid grows (table 1), which is expected. The integral scale was also computed by other means, e.g. integrating the auto-correlation to the first zero-crossing, but this approach was found to be less robust. Kozul et al. (2020, figure 7) demonstrated that while the finite value of the integral scale in flows like the present one is dependent on the method chosen for estimating it, the trends with evolution time (distance) and turbulence intensity are preserved.

The global anisotropy is also reported in table 1 as $u_{\infty}^{\prime} / v_{\infty}^{\prime}$. A separate two-component measurement campaign was performed to obtain these estimates. In general, the anisotropy 
is between 1.1 and 1.2 and thus similar to what is typically reported in grid turbulence (Lavoie et al. 2007) and lower than the anistropy in some other studies of a similar nature (Sharp et al. 2009; Dogan et al. 2019). In most cases, the anistropy grows slightly with downstream distance, which is a result of the slight flow acceleration. Nonetheless, the positional variation in anistropy is always within $\pm 5 \%$, which is approximately the uncertainty of this quantity. The isotropy itself was not a controlled parameter, and generally increasing the turbulence intensity with active grids comes with a loss of istropy (Hearst \& Lavoie 2015). One should thus consider the present results in light of the anisotropy of the flow, which may also have an influence but was not rigorously controlled.

\section{Evolution of the mean and variance profiles}

Freestream turbulence has previously been shown to influence turbulent boundary layers all the way down to the wall (Castro 1984; Dogan et al. 2016; Hearst et al. 2018). While the majority of earlier studies focused on the influence of FST at a single point, in the present study we demonstrate that the evolution of the FST also plays a significant role. We begin with the mean statistics. In figure 4 the velocity and variance profiles for the four inflow conditions are displayed together for every measurement position, showing the differences between the cases at distinct downstream positions. It can be observed that the velocity profiles all collapse in the viscous sublayer, the buffer layer and the logarithmic region. In the viscous sublayer they follow the relation $U^{+}=y^{+}$, with $U^{+}$ being a function of the streamwise velocity and the friction velocity $U^{+}=U / U_{\tau}$. In the logarithmic region, all profiles agree with the law of the wall. This corresponds to the first three terms in (2.1); the plotted logarithmic region reference line has $\kappa=0.39$ and $C^{+}=4.35$. The only significant deviation between cases and locations is in the region between the logarithmic layer and the freestream. In a canonical TBL this is the wake region, where large-scale mixing leads to a velocity defect (Coles 1956). When subjected to high enough freestream turbulence intensity, the wake region is known to be suppressed (Blair 1983a; Thole \& Bogard 1996; Dogan et al. 2016). The freestream, being turbulent itself, leads to a suppression of the intermittent region that typically separates a canonical TBL from an approximately laminar freestream and replaces it with the inherent uniform intermittency of the FST, resulting in a suppressed wake in the boundary layer velocity profile (Dogan et al. 2016). The same can be observed here as presented in figure 4. Case REF with the lowest turbulence intensity of $u_{0}^{\prime} / U_{0}=3.2 \%$ shows traces of a wake region at $x / M=35$ which grows with the development of the boundary layer; the wake is visible at $x / M=55$ and 95 . This evolution becomes even more apparent when looking at the velocity profiles of a single case at the three streamwise positions plotted together as presented in figure 5; we note that figure 5 does not contain different information from figure 4, but that plotting it in this way is also informative for comparison. DNS data of a fully developed canonical TBL without FST (Sillero, Jiménez \& Moser 2013) at a $R e_{\tau}$ comparable to REF is included in figure 5 for reference. The mean velocity profile of REF and the DNS are in good agreement at our last measurement station. The variance profiles are roughly in good agreement, but the background turbulence in the freestream elevates the fluctuations in outer regions of the boundary layer for the experiment. At $x / M=95$, the intermediate cases, $\mathrm{A}$ and $\mathrm{B}$, also exhibit a wake region in the velocity profile (figures $4 c, 5 b$ ) with turbulence intensities of $3.8 \%$ and $5.0 \%$, respectively, but this is still weaker than the REF case and the DNS. For case B, this trend starts to become visible at $x / M=55$ and $u_{\infty}^{\prime} / U_{\infty}=4.7 \%$. This is remarkably consistent with the limit of $u_{\infty}^{\prime} / U_{\infty}=5.3 \%$ found by Blair (1983a). The present results demonstrate for the first time that even if the wake region is initially suppressed by the FST, it redevelops as the 

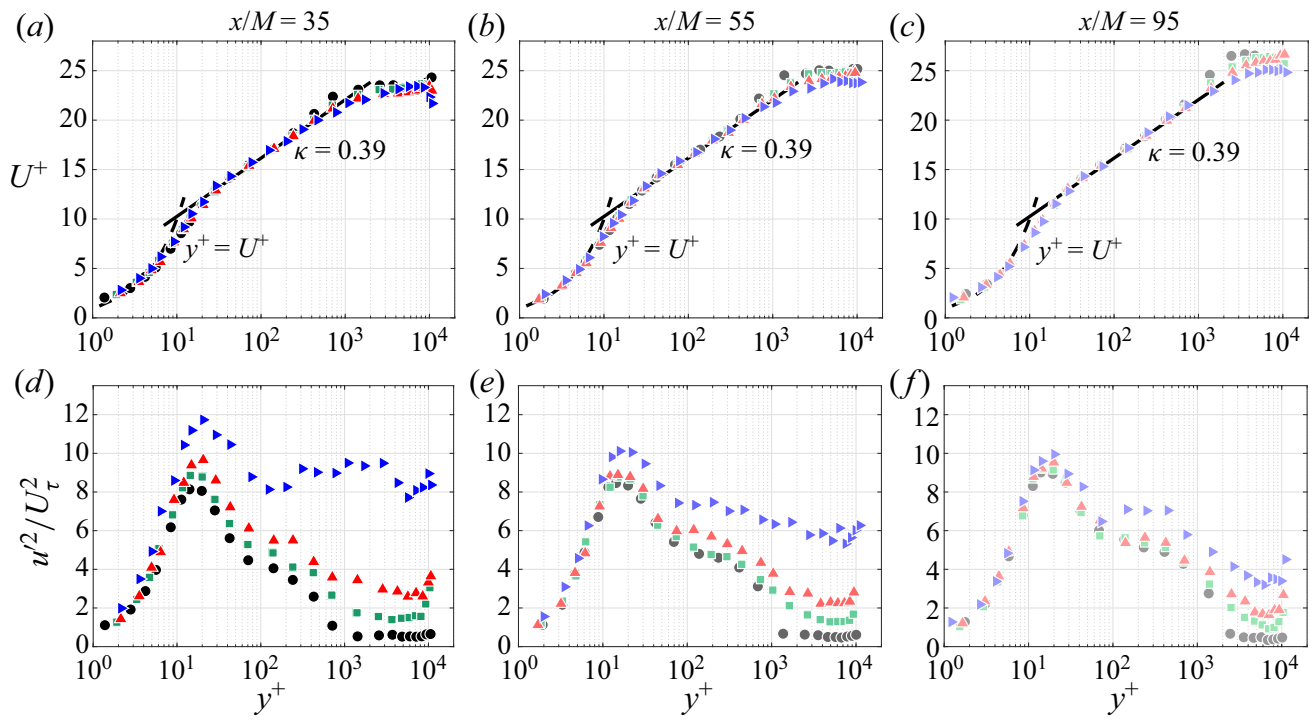

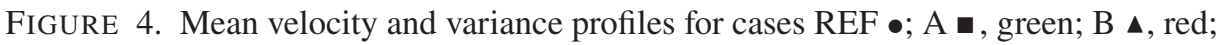
$\mathrm{C} \triangleright$, blue.

FST decays below a certain threshold. This is also supported by looking at Coles' wake parameter $\Pi$ (Coles 1956). He predicted it to be 0.55 for a canonical turbulent boundary layer with no FST. Marusic et al. (2010) confirmed a similar value in their analysis using the model of Perry, Marusic \& Jones (1998). Dogan et al. (2016) found $\Pi=0.55$ in their no-FST case as well and showed that for FST with $7.4 \% \leqslant u_{\infty}^{\prime} / U_{\infty} \leqslant 12.7 \%$ at $x / M=43$, Coles' wake parameter drops to between -0.52 and -0.26 . At $x / M=35$, the present study shows values between -0.57 and -0.08 (table 2). For all cases, $\Pi$ grows with the development of the TBL. The reference case reaches $\Pi=0.37$, which approaches Coles' prediction. Both cases A and B eventually reach positive values for the wake parameter as the wake starts to become visible as one moves downstream. Case $\mathrm{C}$ does not show a visible recovery of the wake, as illustrated in figure 5(c). A visible difference remains compared to the canonical DNS of Sillero et al. (2013). The wake parameter for case $\mathrm{C}$ grows but remains negative and within the range of values for FST found by Dogan et al. (2016) throughout the three positions. $u_{\infty}^{\prime} / U_{\infty}$ does not drop below $7.7 \%$ within the studied distance from the grid for case $\mathrm{C}$, suggesting it does not drop below the required threshold for wake recovery.

In the present study, we define the boundary layer thickness $\delta$ as the point where the velocity reaches $99 \%$ of the freestream velocity, $\delta=\delta_{99}$. For all cases an increase of the boundary layer thickness is observed with the streamwise evolution of the TBL as documented in table $2 . \delta$ at $x / M=35$ also scales with $u_{\infty}^{\prime} / U_{\infty}$, likely due to enhanced mixing. It is also worth highlighting that $L_{u, \infty}$ grows with $u_{\infty}^{\prime} / U_{\infty}$ at $x / M=35$. From the first measurement station, the boundary layers with elevated FST (i.e. cases A, B and C) all grow more rapidly than the REF case.

Freestream turbulence is found to increase the friction velocity $U_{\tau}$ at a given point, in agreement with earlier works (Hancock \& Bradshaw 1989; Blair 1983a; Castro 1984; Stefes \& Fernholz 2004; Dogan et al. 2016; Esteban et al. 2017). This stems from the FST penetrating the boundary layer, increasing mixing and thus the momentum flux towards the wall. This increases the steepness of the velocity profile close to the wall (Dogan et al. 2016) and as a result also the skin friction (Stefes \& Fernholz 2004). 
(a)

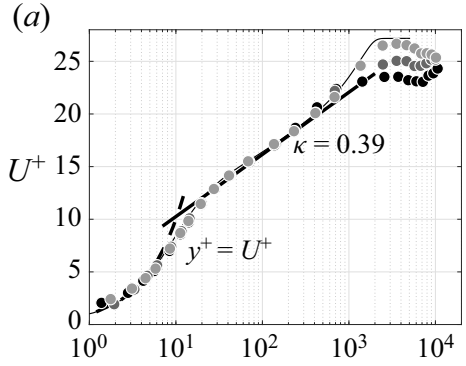

(b)

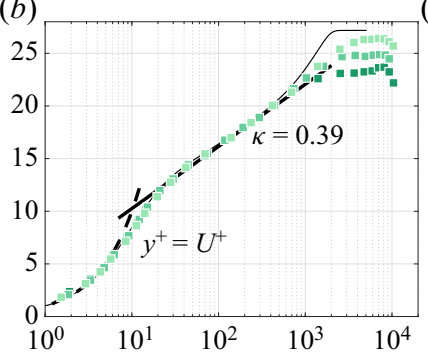

(c)
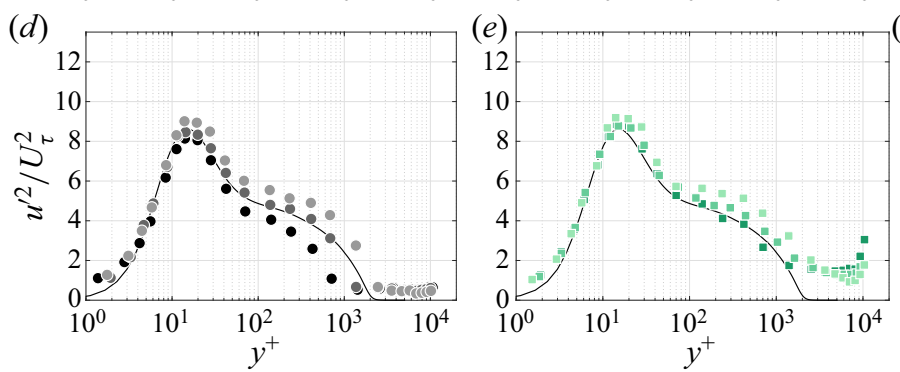
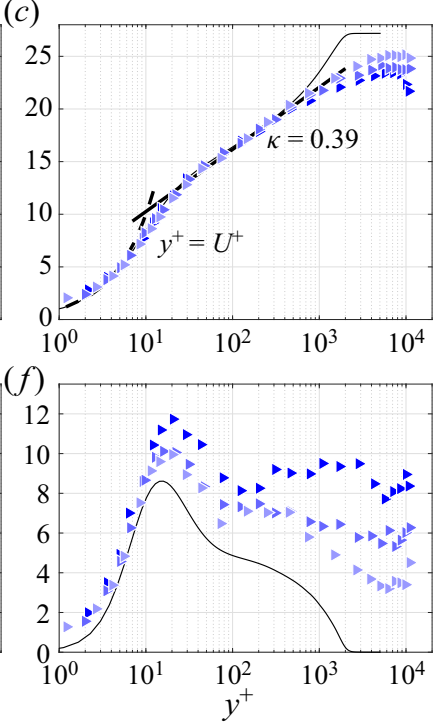

FIGURE 5. Development of mean velocity and variance profiles for cases REF •; A $\bullet$, green and $\mathrm{C} \vee$, blue with fading colours indicating increasing streamwise distance from the grid. DNS data of a fully developed canonical TBL at $R e_{\tau} \approx 1990$ by Sillero et al. (2013) plotted as a reference solid black line.

\begin{tabular}{|c|c|c|c|c|c|c|c|c|c|c|c|c|}
\hline Case & $\begin{array}{c}u_{\infty}^{\prime} / U_{\infty} \\
(\%)\end{array}$ & $x / M$ & $\begin{array}{c}\delta \\
(\mathrm{mm})\end{array}$ & $\begin{array}{c}\delta^{*} \\
(\mathrm{~mm})\end{array}$ & $\begin{array}{c}\theta \\
(\mathrm{mm})\end{array}$ & $H$ & $\begin{array}{c}U_{\tau} \\
\left(\mathrm{mm} \mathrm{s}^{-1}\right)\end{array}$ & $R e_{\tau}$ & $\operatorname{Re}_{\theta}$ & $\Pi$ & $\beta$ & Symbol \\
\hline REF & $\begin{array}{l}3.2 \\
2.9 \\
2.5\end{array}$ & $\begin{array}{l}35 \\
55 \\
95\end{array}$ & $\begin{array}{r}85 \\
95 \\
138\end{array}$ & $\begin{array}{l}12 \\
17 \\
25\end{array}$ & $\begin{array}{c}9 \\
12 \\
19\end{array}$ & $\begin{array}{l}1.31 \\
1.34 \\
1.34\end{array}$ & $\begin{array}{l}14.0 \\
13.5 \\
13.1\end{array}$ & $\begin{array}{c}1210 \\
1310 \\
1870\end{array}$ & $\begin{array}{l}3080 \\
4280 \\
6860\end{array}$ & $\begin{array}{r}-0.08 \\
0.04 \\
0.37\end{array}$ & $\begin{array}{l}0.73 \\
0.64 \\
0.58\end{array}$ & ? \\
\hline A & $\begin{array}{l}5.5 \\
4.7 \\
3.8\end{array}$ & $\begin{array}{l}35 \\
55 \\
95\end{array}$ & $\begin{array}{l}142 \\
170 \\
265\end{array}$ & $\begin{array}{l}16 \\
20 \\
31\end{array}$ & $\begin{array}{l}13 \\
16 \\
24\end{array}$ & $\begin{array}{l}1.24 \\
1.26 \\
1.28\end{array}$ & $\begin{array}{l}14.4 \\
13.8 \\
13.3\end{array}$ & $\begin{array}{l}1990 \\
2490 \\
3700\end{array}$ & $\begin{array}{l}4170 \\
5860 \\
8990\end{array}$ & $\begin{array}{r}-0.19 \\
0.04 \\
0.17\end{array}$ & $\begin{array}{l}1.34 \\
1.13 \\
0.97\end{array}$ & 口 \\
\hline B & $\begin{array}{l}7.4 \\
6.0 \\
5.0\end{array}$ & $\begin{array}{l}35 \\
55 \\
95\end{array}$ & $\begin{array}{l}152 \\
220 \\
308\end{array}$ & $\begin{array}{l}15 \\
21 \\
31\end{array}$ & $\begin{array}{l}12 \\
17 \\
25\end{array}$ & $\begin{array}{l}1.23 \\
1.23 \\
1.26\end{array}$ & $\begin{array}{l}14.8 \\
14.0 \\
13.4\end{array}$ & $\begin{array}{l}2150 \\
3260 \\
4340\end{array}$ & $\begin{array}{l}3840 \\
6230 \\
9050\end{array}$ & $\begin{array}{r}-0.35 \\
-0.18 \\
0.01\end{array}$ & $\begin{array}{l}1.63 \\
1.41 \\
1.23\end{array}$ & $\begin{array}{l}\Delta \\
\Delta\end{array}$ \\
\hline $\mathrm{C}$ & $\begin{array}{r}12.5 \\
9.6 \\
7.7\end{array}$ & $\begin{array}{l}35 \\
55 \\
95\end{array}$ & $\begin{array}{l}246 \\
298 \\
343\end{array}$ & $\begin{array}{l}22 \\
23 \\
29\end{array}$ & $\begin{array}{l}18 \\
19 \\
24\end{array}$ & $\begin{array}{l}1.18 \\
1.21 \\
1.22\end{array}$ & $\begin{array}{l}14.9 \\
14.6 \\
14.2\end{array}$ & $\begin{array}{l}3610 \\
4590 \\
5060\end{array}$ & $\begin{array}{l}6340 \\
7000 \\
8820\end{array}$ & $\begin{array}{l}-0.57 \\
-0.35 \\
-0.26\end{array}$ & $\begin{array}{l}3.09 \\
2.22 \\
1.62\end{array}$ & $>$ \\
\hline
\end{tabular}

TABLE 2. Boundary layer parameters of the test cases at the different streamwise positions.

A decrease in $U_{\tau}$ is observed as the boundary layer develops for each case. This agrees with the behaviour known for spatially evolving canonical turbulent boundary layers without FST (Anderson 2010; Vincenti et al. 2013; Marusic et al. 2015). Values for the friction Reynolds number $R e_{\tau}$ range from 1210 to 5060 and increase both with freestream turbulence intensity and streamwise development. The same is true for $\operatorname{Re}_{\theta}$, with values between 3080 and 9050. The empirical parameter $\beta$ defined by Hancock \& Bradshaw 
(1989) is included in table 2. It follows the same trends as $u_{\infty}^{\prime} / U_{\infty}$, showing that the influence of the FST is dominant in this flow. Greater discussion of this parameter can be found in appendix $\mathrm{C}$.

The variance profiles at the first measurement positions in figure $4(d)$ resemble results from Dogan et al. (2016), Hearst et al. (2018) and You \& Zaki (2019). They showed that the magnitude of the near-wall peak in the variance profiles correlates with the freestream turbulence intensity. The same can be observed in this study. The higher $u_{\infty}^{\prime} / U_{\infty}$, the stronger the near-wall variance peak. FST penetrates the boundary layer and amplifies the fluctuations close to the wall. Moving downstream we can see that the magnitude of the near-wall peaks approach each other until they approximately collapse at $x / M=95$ (figure $4 f$ ). Note that the four flows all still have distinct $u_{\infty}^{\prime} / U_{\infty}, L_{u, \infty}$ and $\delta$ at $x / M=95$. Thus, the present results demonstrate that if the boundary layer is allowed to evolve for a sufficient time, the correlation between the FST magnitude and the near-wall variance peak magnitude diminishes. This differs from earlier measurements performed at a single downstream position that could not observe this phenomenon. Taking a closer look at the development of the near-wall peak for the cases REF, A and C in figure 5, it becomes apparent that the approach to a common near-wall variance peak magnitude is due to different underlying trends in the four cases. For REF, the near-wall variance peak steadily increases with downstream position. This is in agreement with the results from Marusic et al. (2015) for spatially evolving canonical TBLs without FST. This trend is diminished but still present for case A; case B is similar to case A and is not plotted to reduce clutter. For case $\mathrm{C}$, with the highest initial turbulence intensity, the trend reverses: instead of an increase, the near-wall variance peak decreases significantly with the development of the boundary layer. It can be concluded that the spatial development of the near-wall variance peak is strongly dependent on the initial level of turbulence intensity but approaches a common value downstream independently of the initial freestream state, at least for a given $R e_{\tau}$. Hutchins \& Marusic (2007) predicted this to be between 8.4 and 9.2 for the $R e_{\tau}$ examined here. The present measurements find a similar value of $u^{\prime 2} / U_{\tau}^{2} \approx 9.5$. This is slightly higher than what was found by Hutchins \& Marusic (2007), which could be a result of the remaining freestream turbulence still present at the last measurement position, or differences in the noise floors of the measurement techniques used.

The displacement thickness $\delta^{*}=\int_{0}^{\infty}\left(1-U(y) / U_{\infty}\right) \mathrm{d} y$ and momentum thickness $\theta=$ $\int_{0}^{\infty} U(y) / U_{\infty}\left(1-U(y) / U_{\infty}\right) \mathrm{d} y$ grow with streamwise evolution for all cases. The ratio between the two is the shape factor $H=\delta^{*} / \theta$, which is an indicator of the fullness of the boundary layer profile. Small deviations for the dimensional quantities $\delta^{*}$ and $\theta$ can be explained by differences in the mean velocity and uncertainty in the measurements. The trend is still captured accurately. Consequently, in the nondimensional $H$, the small deviations vanish. This study shows that freestream turbulence reduces the shape factor as the boundary layer profile becomes fuller - i.e. the velocity rises more steeply close to the wall, while farther away from the wall the velocity profile becomes flatter. This is in good agreement with previous studies (Hancock \& Bradshaw 1983; Castro 1984; Stefes \& Fernholz 2004; Dogan et al. 2016; Hearst et al. 2018). As presented in figure 6 and table 2, the higher the initial turbulence intensity, the lower the shape factor. For a canonical turbulent boundary layer, Monkewitz, Chauhan \& Nagib (2008) found that the shape factor decreases with increasing $R e_{\theta}$. This is confirmed for each downstream position in this study as depicted in figure 6; the data from Dogan et al. (2016) have also been plotted showing the same trend.

The aforementioned trend pertains to a single position. However, the question of how the evolution of $H$ is impacted by the FST is still open. The data of Hancock \& Bradshaw (1983) suggest a decrease of the shape factor as one moves downstream; this data is also 


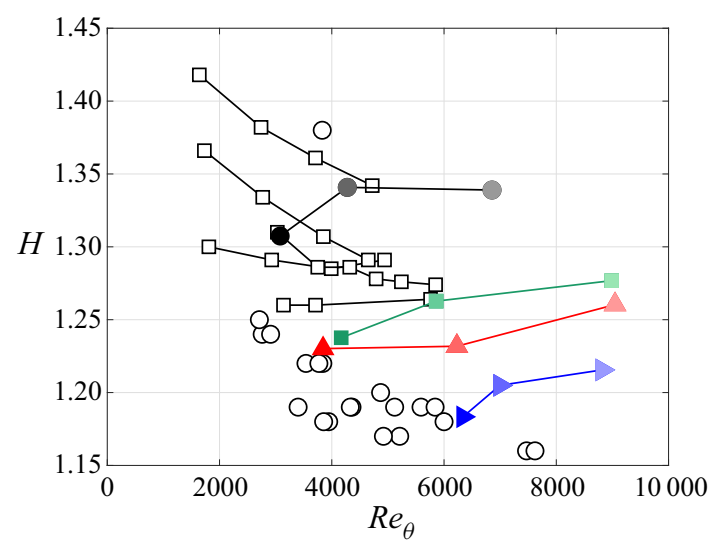

FIGURE 6. Development of the shape factor $H$ for cases REF $\bullet$; A $\bullet$, green; B $\boldsymbol{\Delta}$, red; C $\bullet$, blue with fading colours indicating increasing streamwise distance from the grid. The data of Hancock \& Bradshaw (1983) $\square$ and Dogan et al. (2016) $\circ$ are also included for reference. Lines connecting points indicate that they were acquired from the same set-up but at different streamwise positions. All Dogan et al. (2016) measurements were conducted at the same location but with different freestream conditions.

included in figure 6. It has to be kept in mind that their measurements were for relatively low turbulence intensities, and some of them were very close to the grid. We show that when the turbulence intensity in the freestream is increased further and the measurements are taken past $x / M=30$, this trend reverses. The shape factor is reduced significantly at the first measurement position, and as the freestream turbulence decreases it recovers towards its natural value. This value can be obtained by looking at the shape factor of canonical zero pressure gradient turbulent boundary layers for a wide range of $R e_{\delta^{*}}=$ $U_{\infty} \delta^{*} / v$ as presented by Chauhan et al. (2009). For $R_{\delta^{*}}$ between 4000 and 10000 , as found in the present study, a shape factor between 1.35 and 1.41 would be expected without the presence of freestream turbulence (Chauhan et al. 2009). While the shape factors of Hancock \& Bradshaw (1983) drop away from the canonical values with increasing distance from the grid (Chauhan et al. 2009), the data presented herein trend toward the predicted values. The boundary layer appears to forget it started with different conditions as the influence of these conditions diminishes farther downstream.

The continuous streamwise development of the boundary layer results in an increase of $R e_{\tau}$ for all cases. At the same time $R e_{\tau}$ scales with the level of freestream turbulence which decays with streamwise evolution of the flow. It is therefore interesting to compare boundary layers with similar $R e_{\tau}$ but different paths to get there. This is done in figure 7 with the reference case at $x / M=95$ with $u_{\infty}^{\prime} / U_{\infty}=2.5 \%$ and $R e_{\tau}=1870$ and case A at $x / M=35$ with $u_{\infty}^{\prime} / U_{\infty}=5.5 \%$ and $R e_{\tau}=1990$ (figure $7 a, c$ ), as well as with case A at $x / M=95$ with $u_{\infty}^{\prime} / U_{\infty}=3.8 \%$ and $R e_{\tau}=3700$ and case $\mathrm{C}$ at $x / M=35$ with $u_{\infty}^{\prime} / U_{\infty}=12.5 \%$ and $\operatorname{Re}_{\tau}=3610$ (figure $7 b, d$ ). For the first comparison (figure $7 a, c$ ) with a moderate difference in freestream turbulence intensity, the deviations in the variance profiles are small. Nevertheless, a distinction in the outer region is visible in the velocity profile. Whereas for case A at $x / M=35$ the wake is still suppressed, for the most part, the reference case at $x / M=95$ displays a pronounced wake region. This is particularly interesting given these two cases have essentially the same freestream integral scale, $L_{u, \infty} \approx 310 \mathrm{~mm}$ and $2.1 \leq L_{u, \infty} / \delta \leq 2.3$, suggesting that this parameter is not what is driving the difference in the outer region. When comparing cases with a bigger difference 
(a)
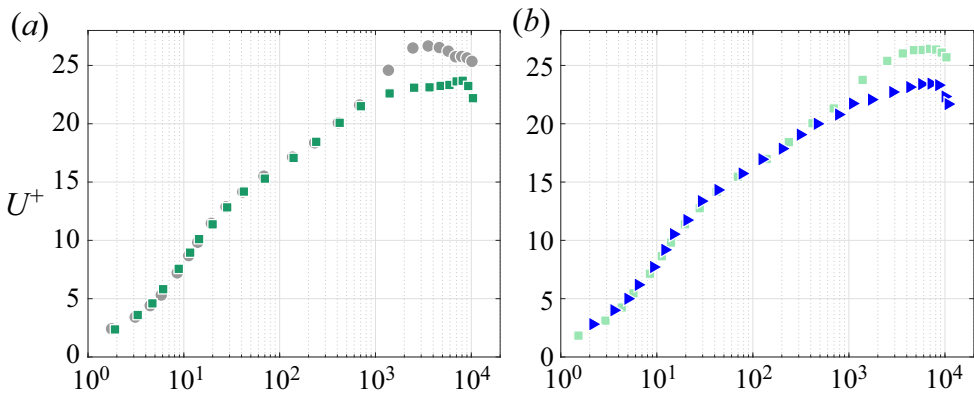

(c)

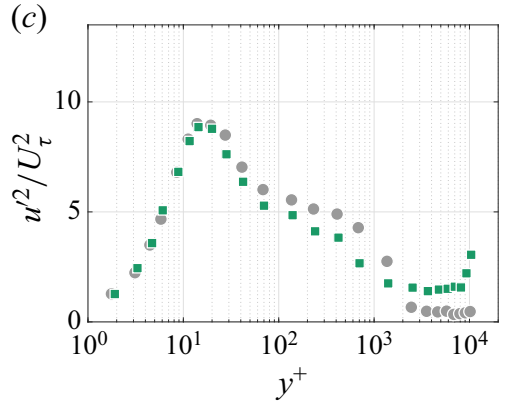

$(d)$

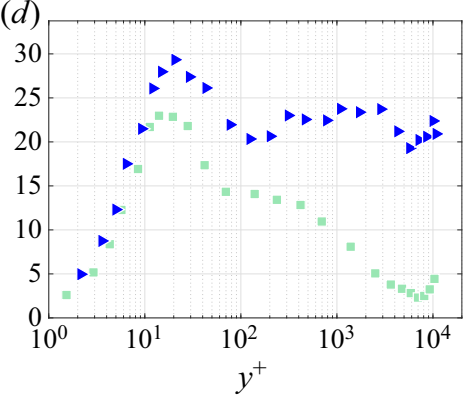

FIgURE 7. Comparison of TBL profiles with similar $R e_{\tau}:(a, c)$ case REF at $x / M=95 \bullet$, grey and case $\mathrm{A}$ at $x / M=35 \mathrm{~m}$, green; $(b, d)$ case $\mathrm{A}$ at $x / M=95 \boldsymbol{m}$, light green and case $\mathrm{C}$ at $x / M=35 \vee$, blue.

in freestream turbulence (figure $7 b, d$ ), the differences become even more distinct. Once again the velocity profiles are collapsed in the viscous sublayer, the buffer layer and the logarithmic region. Farther away from the wall the profiles diverge. For case $\mathrm{C}$ the wake region is fully suppressed at this point, whereas case A at $x / M=95$ shows the reemergence of a wake. In the variance profiles the considerable difference in $u_{\infty}^{\prime} / U_{\infty}$ is visible. Moving closer to the wall it becomes evident that the turbulence intensity in the freestream also influences the boundary layer close to the wall. The near-wall variance peak is significantly more pronounced for the case with the higher freestream turbulence intensity. These particular cases have the same $L_{u, \infty}$ and $1.9 \leq L_{u, \infty} / \delta \leq 2.0$, again suggesting the above differences are not a result of a difference in the size of the large scales in the freestream. The same general trends were also observed at $R e_{\tau} \approx 4500$. One can thus conclude that $R e_{\tau}$ alone is not sufficient to describe the profile of a turbulent boundary layer subjected to FST, but rather $u_{\infty}^{\prime} / U_{\infty}$ and the evolution distance must also be considered at a minimum.

\section{Evolution of the spectral distribution of energy}

Further insight into the processes governing the evolution of a TBL subjected to FST can be gained by looking at the spectral distribution of energy at different streamwise positions. For this, the pre-multiplied spectra, $\phi^{+}=k_{x} \phi_{u} / U_{\tau}^{2}$, at every wall-normal position are plotted together in a contour map illustrating regions and wavelengths, $\zeta^{+}=2 \pi U_{\tau} / k_{x} \nu$, with high and low energy. This is based on the streamwise energy spectra $\phi_{u}$ in normalized wavenumber space $k_{x}$. Computing spectra from the LDV measurements is not as straightforward as it is from hot-wires, which is the more common measurement technique in TBLs. As stated in $\S 2$, we have used the sample 
and hold technique to compute the spectra and applied a bandwidth moving filter. The spectra are also computed over less boundary layer turn-overs than is typical in hot-wire measurements, despite the long sample times used herein. As such, we provide the present spectra as qualitative relative comparisons in which we have confidence, rather than exact quantitative comparisons to the hot-wire-acquired spectra in the literature.

Hutchins \& Marusic (2007) showed that in a canonical turbulent boundary layer there is a fixed peak close to the wall at $y^{+} \approx 15$ and $\zeta^{+} \approx 1000$. They further showed that for high $R e_{\tau}=7300$, an outer spectral peak emerges. The evolution of the spectrograms in a spatially developing TBL for different initial freestream turbulence intensities is presented in figure 8. The first observation is that in agreement with Dogan et al. (2016), Hearst et al. (2018) and Ganapathisubramani (2018), the location of the near-wall spectral peak is independent of the level of freestream turbulence and coincides with the location found by Hutchins \& Marusic (2007). It seems that the small scales close to the wall are not affected by the freestream turbulence. This is displayed explicitly in figure 9 , where the larger scales deviate visibly for the higher FST cases above $u_{\infty}^{\prime} / U_{\infty} \approx 6 \%$, in agreement with Hearst et al. (2018).

Looking at the first measurement position, $x / M=35$, in figure 8 confirms the findings of Sharp et al. (2009), Dogan et al. (2016) and Hearst et al. (2018) that when subjected to strong enough FST an outer spectral peak forms at considerably lower Reynolds numbers than in canonical TBLs - here at $R e_{\tau}=3610$ for case C. For the lowest $R e_{\tau}$ of 1210 , corresponding to the reference case at $x / M=35$, no outer peak exists, and the spectrogram resembles the shape found by Hutchins \& Marusic (2007) for $R e_{\tau}=1010$. Cases $\mathrm{B}$ and $\mathrm{C}$ at $x / M=35$ demonstrate a timid emergence of an outer spectral peak. The novel element of the present study is the streamwise development of these features. For cases REF, A and B, with initial turbulence intensities between $3.2 \%$ and $7.4 \%$, the outer spectral peak grows in magnitude and moves away from the wall as the boundary layer develops. Of these three cases, case B with the highest initial turbulence intensity $u_{0}^{\prime} / U_{0}$, shows the strongest outer spectral peak. This agrees with the trend for increasing $R e_{\tau}$ detected by Hutchins \& Marusic (2007) in a canonical TBL.

Up until the present study there has been no reason not to expect a growth of the outer spectral peak with increasing $R e_{\tau}$ for higher freestream turbulence intensities as well. Instead, case $\mathrm{C}$ with the highest initial turbulence intensity of $u_{0}^{\prime} / U_{0}=12.5 \%$, presents different behaviour. The outer spectral peak is pronounced at $x / M=35$. In contrast to the expected continuous growth of the outer spectral peak in canonical TBLs, here it gradually decreases as the boundary layer develops and the freestream turbulence decays. Thus, if one did not know the measured values of $R e_{\tau}$, the spectrogram from earlier in the spatial evolution of case $\mathrm{C}$ gives the impression it is at a higher $R e_{\tau}$ than those from farther downstream. In contrast to the lower FST cases, the decay of the freestream turbulence more significantly influences the spectrogram than the growth of the TBL. This fading of the outer spectral peak is visible throughout the three measurement positions for case $\mathrm{C}$. This behavior becomes more evident when looking at the net change $\Delta^{+}=$ $\left(\phi^{+}-\phi_{0}^{+}\right) / \phi_{0, \text { max }}^{+}$in spectrograms, where $\phi_{0}^{+}$is the spectrogram at $x / M=35$. This is displayed in figure 10 for the reference case compared to case $C$ with the highest freestream turbulence intensity. The reference case (figure $10 a, b$ ) shows the slow emergence of an outer peak with a positive net change $\Delta^{+}$for $\zeta^{+} \approx 10^{4}$ most distinctly in the outer regions of the boundary layer at $y^{+} \approx 10^{3}$. The opposite is observed for case $\mathrm{C}$ in figure $10(c, d)$, with a negative net change where the outer spectral peak was initially most pronounced at $10^{3} \lesssim y^{+} \lesssim 10^{4}$ and $10^{4} \lesssim \zeta^{+} \lesssim 10^{5}$. The location of the outer spectral peak in outer scaling, i.e. $y / \delta$ and $\zeta / \delta$, does not coincide with the location for canonical TBLs identified by Hutchins \& Marusic (2007). This is to be expected for a TBL subjected to FST (Dogan 


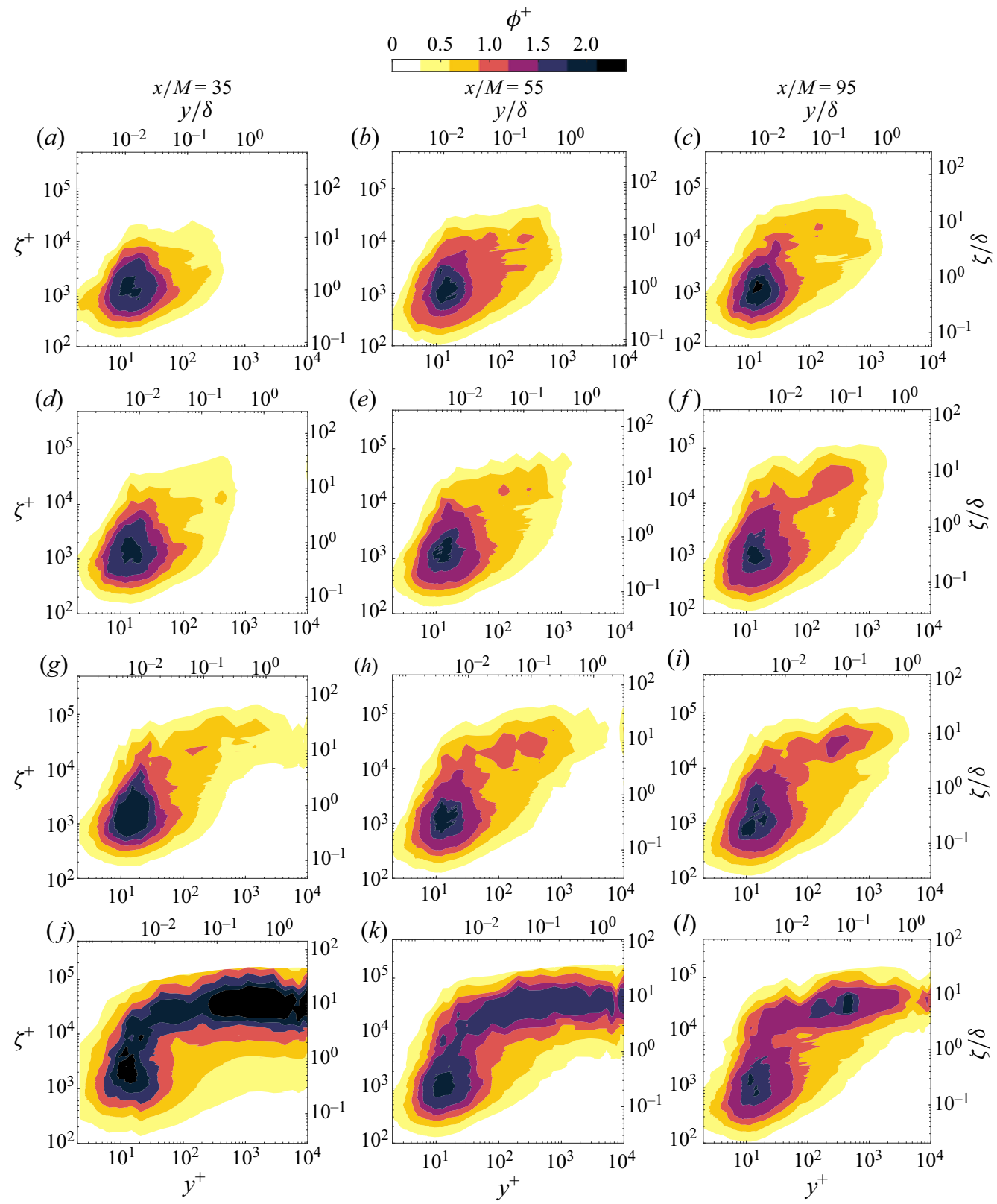

FIgURE 8. Spectrograms for cases REF $(a-c)$, A $(d-f), \mathrm{B}(g-i)$ and C $(j-l)$ at the three streamwise positions with increasing level of freestream turbulence from top to bottom.

et al. 2016; Hearst et al. 2018). The reason for this is that the peak is superimposed onto the outer boundary layer by the freestream turbulence. In fact, the peak is situated much higher for the FST cases and moves only once the boundary layer starts to redistribute the energy. This is documented in great detail for numerous cases in Hearst et al. (2018). As the outer peak evolves in this study, it approaches $\zeta_{x} / \delta \approx 10$ and $y / \delta \approx 0.4$ as found by Hearst et al. (2018). 


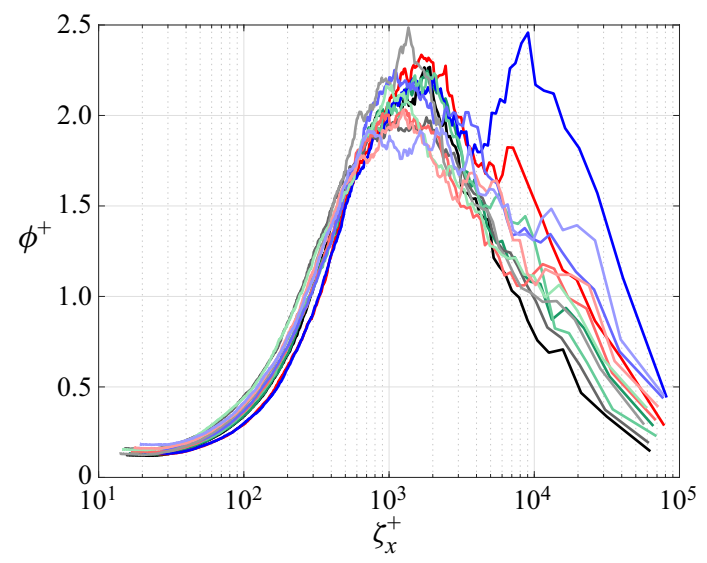

FIGURE 9. Normalized pre-multiplied velocity spectra at the near-wall spectral peak for cases REF solid black line, A solid green line, B solid red line, C solid blue with fading colours indicating increasing streamwise distance from the grid.

It is also interesting to compare case $\mathrm{B}$ at $x / M=35$ (figure $8 g$ ) and case $\mathrm{C}$ at $x / M=95$ (figure $8 l$ ), which have approximately the same freestream turbulence intensity $7.4 \% \leq u_{\infty}^{\prime} / U_{\infty} \leq 7.7 \%$ and integral scale relative to the boundary layer thickness $2.6 \leq$ $L_{u, \infty} / \delta \leq 2$.7. Their spectrograms look very different, demonstrating the importance of the evolution on the energy distribution within the boundary layer. Furthermore, when comparing cases with similar $R e_{\tau}$, e.g. case $\mathrm{A}$ at $x / M=95$ (figure $8 f$ ) and case $\mathrm{C}$ at $x / M=35$ (figure $8 j$ ), the difference is even more apparent. Figure $8(f)$ shows a hint of an outer spectral peak, while figure $8(j)$ represents the most prominent occurrence of an outer peak of all the measurements. This underlines the fact that $R e_{\tau}$ must be considered alongside $u_{\infty}^{\prime} / U_{\infty}$ and the evolution distance when studying TBLs subjected to FST.

\section{Global trends}

The way this experiment was constituted, there were two main factors modulating the boundary layer contrarily to each other. On the one hand, the TBL was evolving spatially, growing and becoming more developed. On the other hand, the FST, which artificially matured the state of evolution of the boundary layer, decayed with increasing distance $x$ from its origin, the active grid. The streamwise evolution of a boundary layer may be expressed through $\operatorname{Re}_{x}=U_{\infty} x / v$. Figure 11 summarizes how the natural growth of the boundary layer and the decay of the freestream turbulence interact, and which prevails under what conditions. The implications for different characteristics of a TBL are examined as the boundary layers evolve spatially.

The boundary layer at a single position thickens with increasing freestream turbulence intensity. As the flow evolves, the turbulence in the freestream decays and the integral scale grows. At the same time the boundary layer develops. Overall this leads to a growth of the boundary layer thickness for all levels of freestream turbulence. Figure 11(b) shows a relatively uniform stacking of the boundary layer thickness with $u_{\infty}^{\prime} / U_{\infty}$ for low $R e_{x}$. As the flow develops, the higher FST intensity cases A, B and C have similar values of $\delta$, while $\delta$ for REF is demonstrably smaller. The influence of $u_{\infty}^{\prime} / U_{\infty}$ on $\delta$ decreases as the flow evolves, but a distinct difference remains between low and moderate to high FST intensity. 

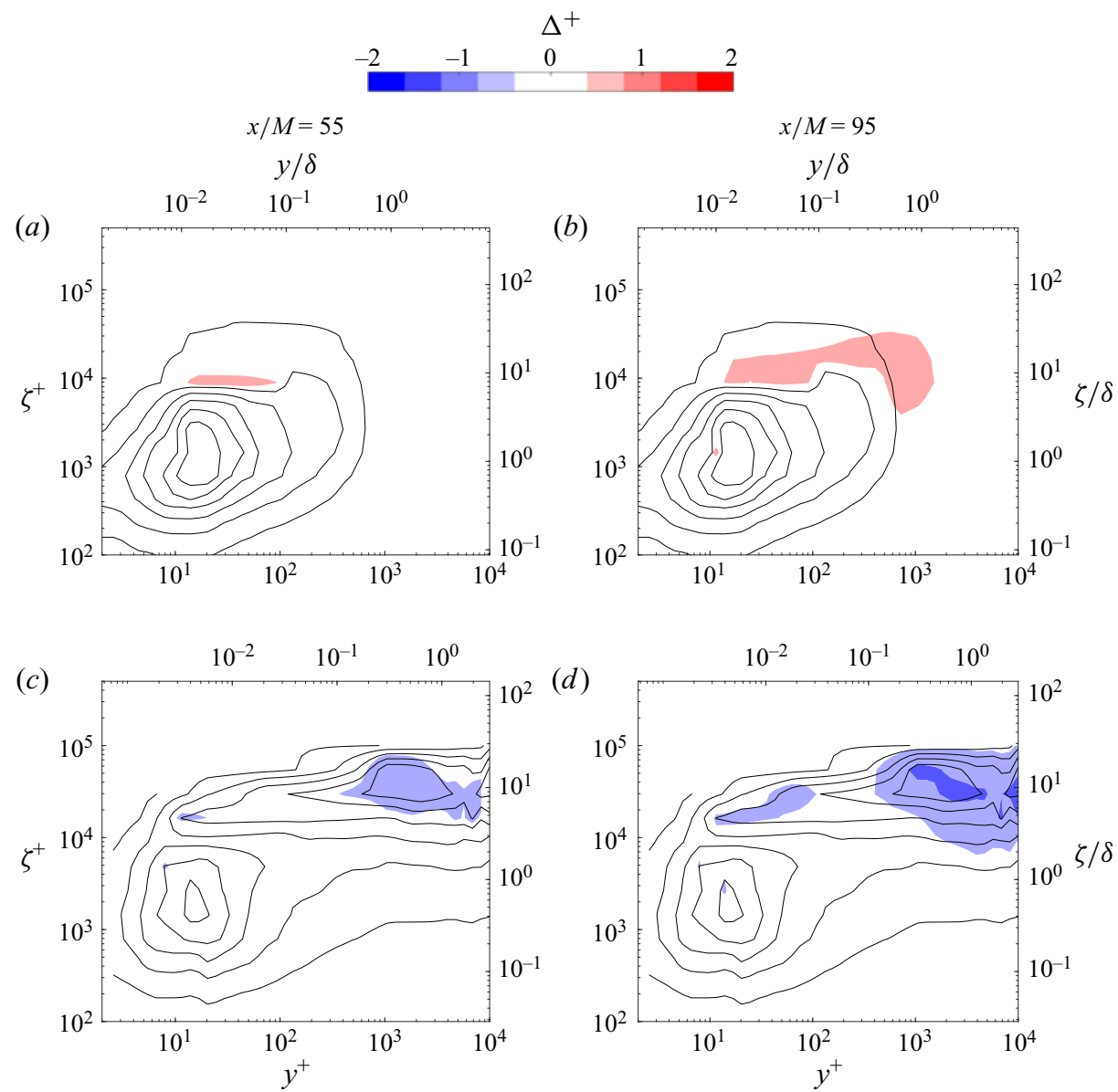

FIGURE 10. Net change $\Delta^{+}=\left(\phi^{+}-\phi_{0}^{+}\right) / \phi_{0, \max }^{+}$in spectrograms at $x / M=55$ and $x / M=$ 95 for cases $\operatorname{REF}(a, b)$ and $\mathrm{C}(c, d)$ with respect to initial spectrogram at $x / M=35$. The contour lines of the initial spectrogram are imprinted as a reference.

For a sufficiently developed canonical turbulent boundary layer, the shape factor $H$ decreases with increasing $R e_{x}$ (Vincenti et al. 2013; Marusic et al. 2015). This decrease can also be achieved by introducing FST in the flow. The result is, contrarily to a canonical TBL, $H$ grows with increasing $R e_{x}$ as the boundary layer develops beneath decaying FST. Presumably there is a turning point when $H$ will start decreasing again. Throughout the examined range, the shape factor remains distinguished by $u_{\infty}^{\prime} / U_{\infty}$ (figure 11c). The influence of the initial difference in freestream turbulence is transported through the examined range of $R e_{x}$. Similar behaviour can be observed for the wake region of the TBL. This is quantified through Coles' wake parameter $\Pi$, which is known to trend towards a fixed value for canonical conditions with high Reynolds numbers and sufficient development length (Marusic et al. 2010). Freestream turbulence suppresses the intermittency in the wake region, thus leading to the suppression of the typical flow profile seen in the wake region and a significantly depleted wake parameter (Dogan et al. 2016). The stronger the freestream turbulence intensity, the lower $\Pi$ becomes. The wake is predominantly influenced by the FST, and as it decays, the wake 

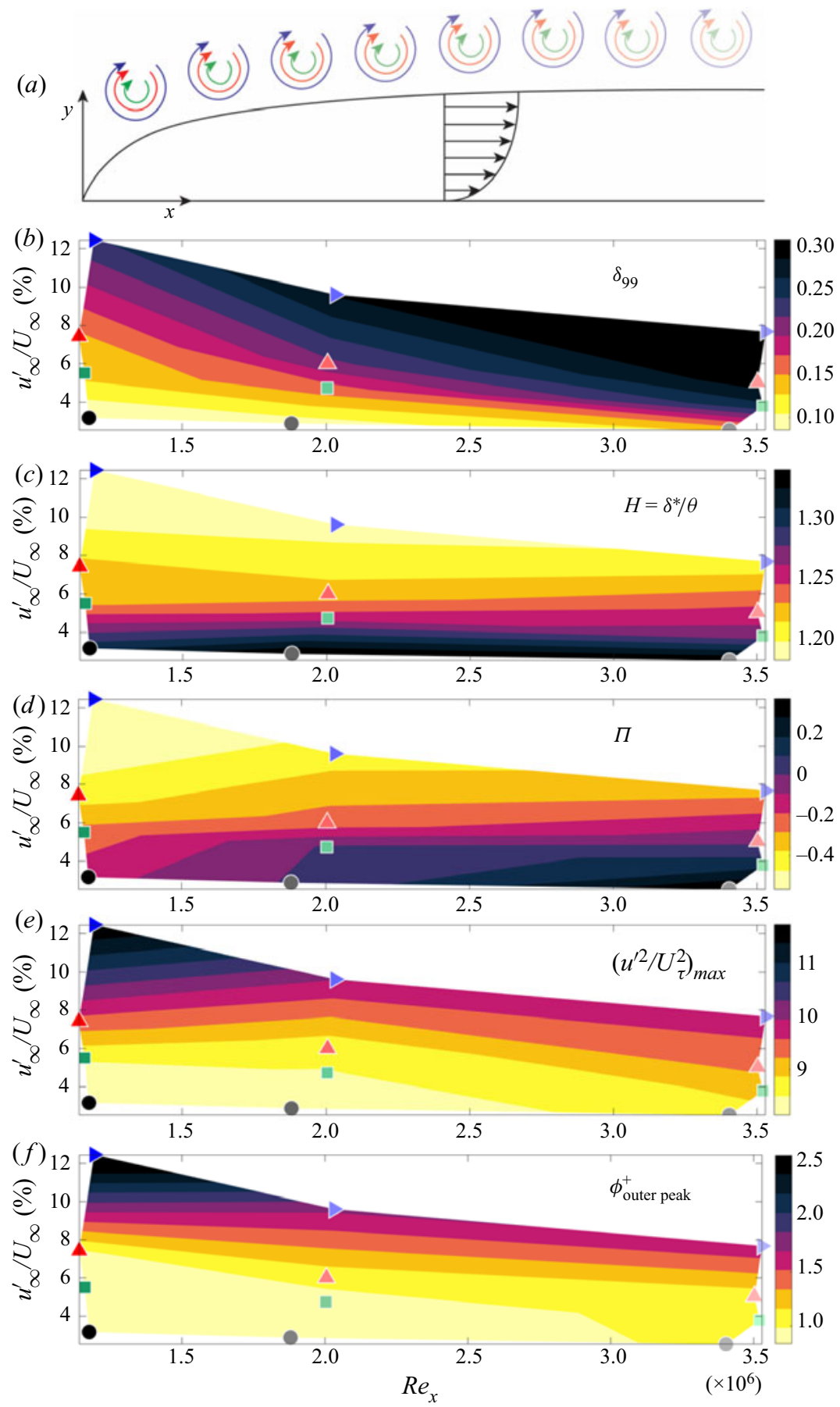

FIGURE 11. Trends for an evolving turbulent boundary layer subjected to different levels of

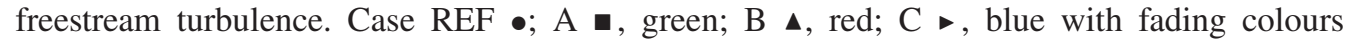
indicating increasing streamwise distance from the grid. 
becomes more pronounced. The overall change of $\Pi$ with spatial evolution is more substantial than the change to $H$. For the lower turbulence intensities, $\Pi$ approaches the analytical value of 0.55 (Coles 1956), and a visible wake region re-emerges within the investigated spatial development range (figure 5). The change in shape of the boundary layer indicates that the FST penetrates the boundary layer and has an influence on its evolution.

How deep and how significant that influence is becomes evident when looking at the modulation of the near-wall variance peak at $y^{+} \approx 15$. The magnitude is strongly dependent on the level of turbulence in the freestream, with a higher turbulence intensity correlating with a higher peak in the variance. For canonical TBLs, the near-wall peak increases with the evolution of the boundary layer until the profiles become self-similar. This behaviour can be observed for lower initial freestream turbulence up to $u_{0}^{\prime} / U_{0}=5.5 \%$. For the highest freestream turbulence intensity, the decay of the turbulence proves to be dominant, as the near-wall variance peak decreases in magnitude as the flow evolves.

For high enough $R e_{\tau}$, TBLs develop an outer peak in the spectral energy distribution (Hutchins \& Marusic 2007). This state can also be reached by subjecting the boundary layer to high-intensity freestream turbulence (Dogan et al. 2016; Hearst et al. 2018). For canonical TBLs, this peak develops as the boundary layer grows spatially and $R e_{\tau}$ increases. This is observed for the lower freestream turbulence cases $3.2 \% \leqslant u_{0}^{\prime} / U_{0} \leqslant$ $7.4 \%$ here. Initially there is no outer peak visible in the spectrograms, but as the boundary layer develops, the magnitude of the outer peak gradually increases. This evolution looks very different for the highest level of freestream turbulence. A strong peak exists at the first measurement position, then proceeds to decrease with streamwise evolution of the flow. For this case, the decay of the FST appears to drive the phenomenology. The drop in outer peak magnitude is significantly higher than the observed increase for the lower FST cases (figure $11 f$ ). We thus again arrive at the conclusion that these flows must be parameterized by $R e_{\tau}, u_{\infty}^{\prime} / U_{\infty}$ and the streamwise development of the flow.

\section{Conclusions}

The evolution of a turbulent boundary layer subjected to different freestream turbulent flows was studied experimentally for $1210 \leqslant R e_{\tau} \leqslant 5060$. The freestream turbulence was generated with an active grid in a water channel. Boundary layer profiles were taken at three streamwise positions for four inflow turbulence intensities $3.2 \% \leqslant u_{0}^{\prime} / U_{0} \leqslant 12.5 \%$. It is important to appreciate that the conclusions presented herein are derived from the results of the present measurement campaign and the investigated turbulence intensities, integral scales and anisotropy. This is the first in-depth analysis of how freestream turbulence influences the characteristics of a spatially evolving turbulent boundary layer at Reynolds numbers of this magnitude. In particular, the interaction of decaying freestream turbulence with a developing turbulent boundary layer was examined. The main findings of this study are:

(i) The development of the boundary layer mean velocity profile changes in the presence of freestream turbulence. Instead of a decrease in shape factor, as observed in canonical turbulent boundary layers (Monkewitz et al. 2008), $H$ increases as the freestream turbulence decays. The suppression of the wake region for high freestream turbulence intensities observed in accordance with Blair (1983a), Thole \& Bogard (1996) and Dogan et al. (2016) can be reversed as the flow 


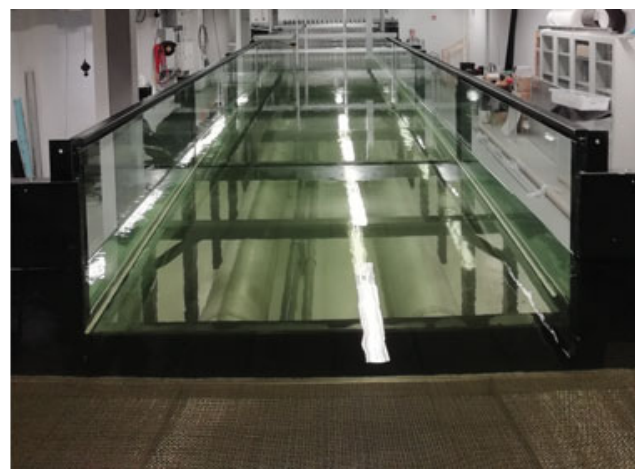

FIGURE 12. Water channel facility viewed from the end tank.

evolves downstream. It was shown that as the freestream turbulence decays below $u_{\infty}^{\prime} \approx 5 \%$, the wake region is recovered.

(ii) The influence of the freestream turbulence on the magnitude of the near-wall variance peak decreases as the freestream turbulence decays in the spatially developing flow. For high-intensity FST cases, a decrease in near-wall variance peak magnitude was observed contrarily to lower freestream turbulence levels where an increase was noted with the development of the boundary layer. The latter is similar to canonical turbulent boundary layers without freestream turbulence.

(iii) Spectral analysis showed that an outer peak in the spectrograms can be formed in two ways, and that this is pivotal for the evolution of the spectrograms. For $u_{0}^{\prime} / U_{0}=3.2-7.4 \%$, it emerges gradually as the boundary layer evolves as observed for canonical boundary layers by Hutchins \& Marusic (2007) and Marusic et al. (2015). The mechanisms at the wall that naturally generate this peak are dominant here. However, an outer spectral peak can also be imprinted by high intensity freestream turbulence (Sharp et al. 2009; Dogan et al. 2016; Hearst et al. 2018). For the latter, it was demonstrated that as the flow develops spatially and the freestream turbulence decays, the outer spectral peak becomes weaker, and hence the flow does not remember that it had an outer peak earlier in its evolution. The information available in the literature does not suggest that the boundary layer would effectively regress to a less mature state once the freestream turbulence decayed, and evidence of this is presented herein for the first time.

Generally, it was found that for turbulent boundary layers subjected to freestream turbulence, the previous perspective that one could parameterize the flow with just a few parameters, i.e. $R e_{\tau}$ or $R e_{\theta}, u_{\infty}^{\prime} / U_{\infty}$ and $L_{u, \infty}$, is incorrect. For example, flows with similar $R e_{\tau}, u_{\infty}^{\prime} / U_{\infty}$ or $L_{u, \infty} / \delta$ can have significantly different boundary layer characteristics depending on the evolution of the freestream turbulence and boundary layer. Thus, the relative evolution of the freestream turbulence and the boundary layer must also be considered.

\section{Funding}

This work was funded by the Research Council of Norway project no. 288046 (WallMix). 


\section{Declaration of interests}

The authors report no conflict of interest.

\section{Appendix A. Water channel facility}

The water channel is a recirculating facility with a capacity of 65 tons of water. A picture of the facility as viewed from the end tank is shown in figure 12 and a schematic was provided in figure 1. It is driven by two Siemens 1AV2186B 3-phase squirrel-cage motors each connected to two counter-rotating propellers. Each motor-pump assembly forms a part of the return pipe system that runs the length of the water channel underneath the test section. The motors are controlled via two ABB ACS550 variable frequency drives. The two return pipes supply water to the channel through a $90^{\circ}$ bend each into a polyethylene settling chamber. The end section of the outlet is constructed from porous sheet metal to provide a diffuse source of water. A flat circular plate is also secured within the porous section to minimize the size of the water jet from the outlet. A large acrylic surface plate with adjustable height is placed above the outlet to dampen the surface waves caused by the water flowing out of the exits. After the outlet, the water flows through a porous plate, followed by a honeycomb and then a pair of stainless steel screens with progressively smaller mesh size for flow conditioning. A $4: 1$ fibreglass contraction connects the settling chamber and the test section. Between the contraction and the test section, there is a slot measuring $200 \mathrm{~mm}$ wide intended for the installation of turbulence generating grids. This section consists of permanently mounted acrylic frames with interchangeable inner skins, allowing for an active grid, passive grid or clean flow. The test section measures $11 \mathrm{~m} \times 1.8 \mathrm{~m} \times 1 \mathrm{~m}$ internally and is constructed from float glass panes supported by stainless steel frames. The maximum water level is $0.8 \mathrm{~m}$. The clear glass construction provides optical access for laser diagnostic measurements and other optically-based measurement techniques. The water exits the test section into a stainless steel end tank, where it recirculates back to the return pipes. A stainless steel frame with wire meshes on both sides is installed in the end tank at an angle. This device acts as a wave energy dissipator to prevent large reflected waves from the end tank. The height and angle of the dissipator are adjustable. The water is kept free from debris and algae through a filter system consisting of a pump, a cyclone filter, a particle filter and a UV-lamp. There is no active temperature control for the water channel; however, once the water reaches an equilibrium with the room temperature, the daily variation in water temperature is less than $0.5^{\circ} \mathrm{C}$, which is monitored with a thermocouple.

The freestream flow velocity is measured through a Höntzsch ZS25 vane wheel flow sensor with an accuracy of $0.01 \mathrm{~m} \mathrm{~s}^{-1}$. The flow sensor has an analogue current output, which is converted to an analogue voltage output and connected to a NI-9125 C series voltage input module. A T-type thermocouple is placed in the test section to measure the water temperature. It is connected to a NI-9210 C series temperature input module. Both modules are plugged into a NI cDAQ-8178 CompactDAQ chassis, which is in turn connected to a data acquisition computer.

\section{Appendix B. Active grid}

An active grid is an instrument for controlling freestream turbulence that is gaining popularity. While active grids are becoming more common, comprehensive documentation of them is still sparse. As such, this section offers a detailed description that can be potentially useful for others in the future. 


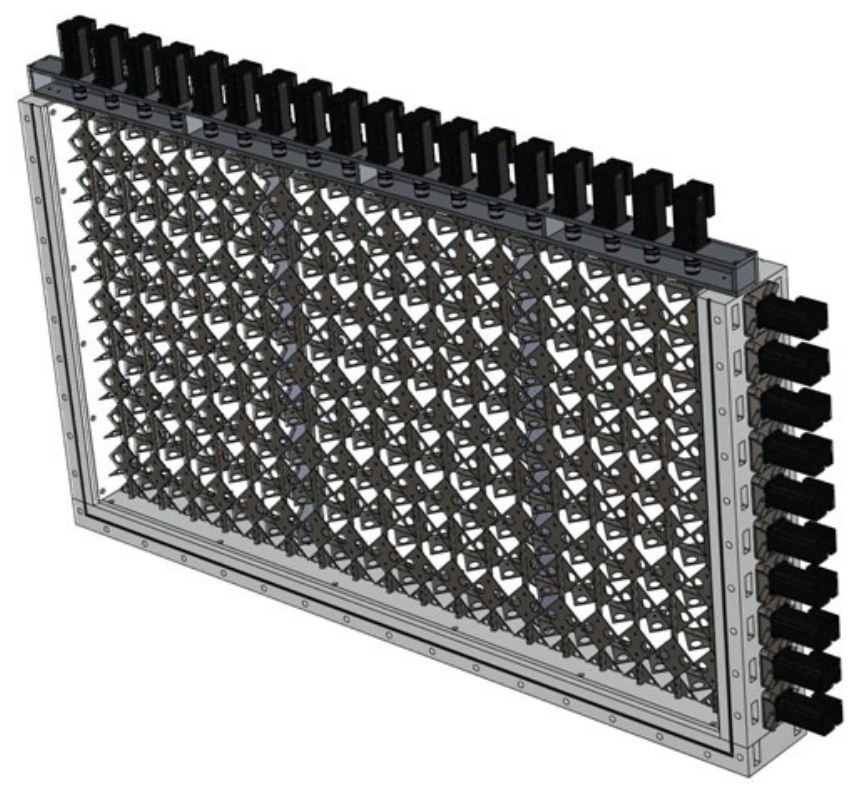

FIGURE 13. Three-dimensional view of the active grid at the water channel facility at the Norwegian University of Science and Technology.

The active grid used in the present study was designed in-house, and a three-dimensional drawing of the design is shown in figure 13. It consists of 28 independently controlled stainless steel rods arranged in a biplanar square mesh, with 10 horizontal rods and 18 vertical rods. The mesh length $M$ defined by the centre to centre distance between the rods is $100 \mathrm{~mm}$, and the rods measure $12 \mathrm{~mm}$ in diameter. The grid stretches across the entire cross-sectional area of the test section. Stainless steel $1 \mathrm{~mm}$ thick square-shaped wings are attached to the rods in a space-filling manner. The sides of the wings measure $70.71 \mathrm{~mm}$, such that the diagonal measures $100 \mathrm{~mm}$, which matches the mesh length of the grid. Each wing has two $24 \mathrm{~mm}$-diameter holes cut out of it in order to reduce the loading on the motors during actuation sequences, as well as to make sure a $100 \%$ blockage scenario is impossible. The maximum blockage ratio achievable by the active grid is $81.9 \%$, and the minimum blockage ratio is $22.6 \%$. The rods are CNC-machined to have a $1 \mathrm{~mm}$ deep flat for wing mounting, such that the wings sit flush with the rod. As the maximum water level is $0.8 \mathrm{~m}$, only the bottom eight horizontal rods are submerged at the maximum capacity; the top two rods are always in the air and are meant for possible future expansion of the facility. Figure 2 shows the middle section of the active grid at maximum blockage. The horizontal rods are supported at four locations by low-friction plastic bushings, two at the ends and two within the grid body, located at the $\frac{1}{3}$ grid width positions from the ends. The vertical rods are secured in place at the ends through the same low-friction plastic bushings at the bottom and through stainless steel bearings at the top. The plastic bushings that support the horizontal rods are inlaid inside CNC-machined acrylic plates that attach to the permanent frame between the contraction and the test section. The plates measure $164 \mathrm{~mm}$ in width and span the entire inner portion of the permanent frame. They are designed to sit flush with the walls of the test section so that the flow downstream is not affected. The plates are also designed to be removable. The vertical rods are secured at the top through bearings mounted inside a custom-designed aluminium frame. The rods are then attached to the motors through a stainless steel flexible coupling 
and a custom-made stainless steel coupling. This coupling is designed to interface between the flexible coupling and the motor, which have different diameter shafts. The coupling is secured onto the motor via set screws. The motors for the vertical rods are secured onto the aluminium frame, which is in turn fastened onto the permanent frame for the active grid. The horizontal rods are connected to the motors through the same mechanism, except for a custom-made water-tight mounting box for the motor. The water-tight box features a custom-designed double lip seal from Trelleborg $\mathrm{AB}$ sandwiched between a plastic bushing and a stainless steel bearing. The bushing is on the wet side of the seal while the bearing is on the dry side. The same custom-made coupling mentioned previously goes through the seal assembly to connect to the motor, and the motor is mounted to the dry side of the water-tight box. Large drainage openings are milled into the water-tight box so that in the event of a leakage, the water would drain away before reaching the motor, thus protecting the motors from water damage. The entire vertical rod assembly, which includes the rods with wings, aluminium frame and motors, is designed to be removable as one unit, while the horizontal rods are designed to be removable from the flexible couplings. The motors for the horizontal rods along with the water-tight boxes are permanently mounted to the side of the frame.

The motors used are STM23S-3RE stepper motors from Moons Ltd. (also marketed as Applied Motion Products in other countries). The motors are equipped with internal encoders and motion controllers. Power is supplied by two TDK-Lambda 48 V 52 A DC power supplies. Each motor can draw up to $2.5 \mathrm{~A}$ at $48 \mathrm{~V}$. The motors are daisy-chained together and connected to a PCI serial adapter card in a computer. Unique ASCII names are given to each motor for identification by the controlling scripts. Control commands are generated and transmitted through MATLAB scripts, which can independently control each motor's rotation speed, acceleration, direction and duration. To the authors' best knowledge, this is the largest active grid installation for a water channel facility in the world at the time of writing.

\section{Appendix C. A note on the empirical parameter of Hancock \& Bradshaw (1983)}

Seminal experimental works on the influence of FST on a TBL from the 1980s placed emphasis on both the turbulence intensity and the integral scale (Hancock \& Bradshaw 1983, 1989; Castro 1984). In fact, Hancock \& Bradshaw (1983) developed an empirical parameter, $\beta=\left(u_{\infty}^{\prime} / U_{\infty}\right) /\left(L_{u, \infty} / \delta+2\right)$, which appeared to correlate well with the wall shear stress and wake in their flows. More recent studies have placed more emphasis on the turbulence intensity - e.g. Dogan et al. (2016); Hearst et al. (2018) - with the latter even proposing that for higher turbulence intensities, the integral scale does not necessarily play a significant role. The primary difference between the earlier studies and contemporary ones is that the recent use of active grids has allowed for high turbulence intensity $(>10 \%)$ to be achieved much farther downstream from the turbulence generating grid $(x / M>30)$. Hearst et al. (2018) noticed that one of the reasons for this result appeared to be that $\delta$ adjusts itself such that the peak in the premultiplied energy spectrum is at approximately $\sim 10 \delta$. This appeared to be true for all 17 of their cases with $7 \% \lesssim u_{\infty}^{\prime} / U_{\infty} \lesssim 13 \%$, see their figure 1(b). This was in fact first noticed by Dogan et al. (2016) in their figure 8, although they did not emphasize it as strongly. This is also approximately true in the present investigation, particularly for the more turbulent cases, as depicted in figure 14 . This relationship suggests that given sufficient coevolution distance, the energy containing scales and the boundary layer height adjust to keep a constant proportionality between them (Hearst et al. 2018). Thus, $L_{u, \infty}$ and $\delta$ are co-dependent, and the ratio $L_{u, \infty} / \delta$ is relatively constant, whilst the turbulence intensity decays, resulting in the appearance 


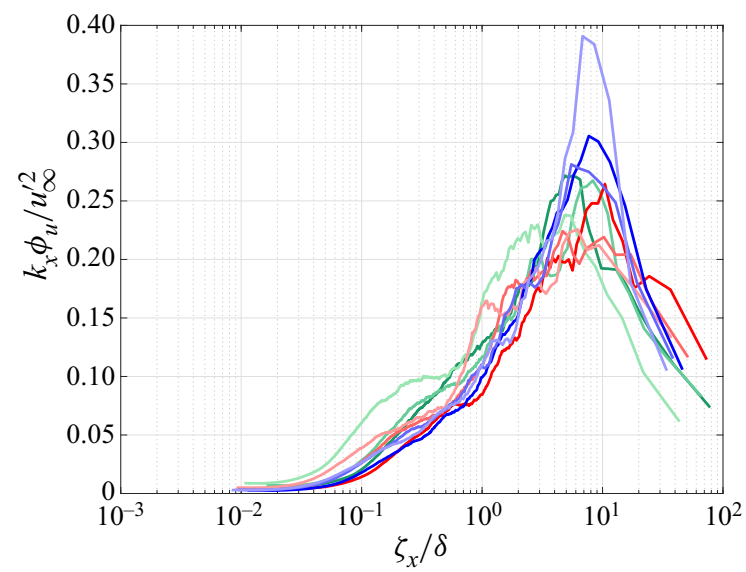

FIGURE 14. Normalized pre-multiplied velocity spectra in the freestream for cases A solid green line, B solid red line, $\mathrm{C}$ solid blue line with fading colours indicating increasing streamwise distance from the grid.
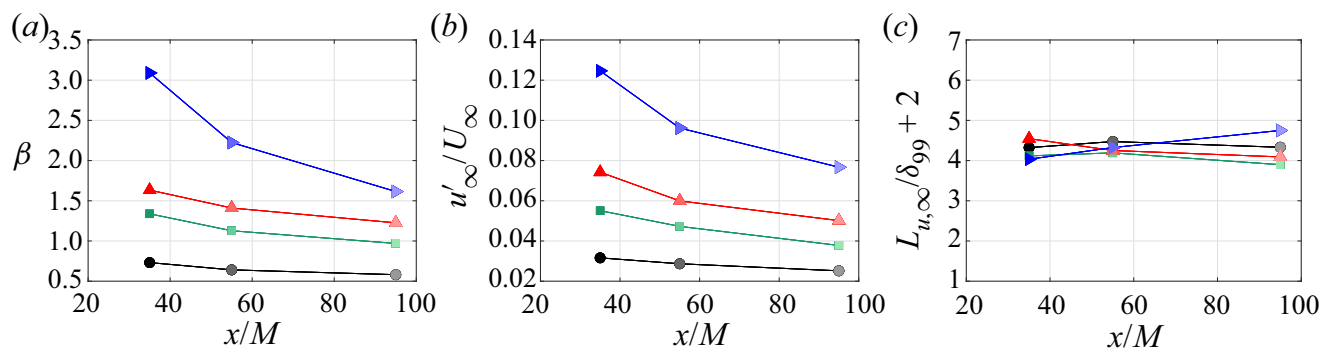

FIGURE 15. Analysis of the empirical parameter $\beta$ introduced by Hancock \& Bradshaw (1983) for cases REF $\bullet$; A $\boldsymbol{\bullet}$, green; B $\boldsymbol{\Delta}$, red; C $\bullet$, blue. Note that the scaling on these figures was chosen to represent equal changes in $\beta$ and the two contributing parameters included in it.

of primary dependence on the turbulence intensity. It is important to acknowledge that although this result has been observed in two different facilities, it is possible that it is a consequence of the integral scales produced in those studies, and that if significantly larger or smaller integral scales were investigated, a different result or trend may emerge.

For completeness, we present $\beta$ along with $u_{\infty}^{\prime} / U_{\infty}$ and $L_{u, \infty} / \delta+2$ in figure 15 , with the $y$-axis scaled to show the same percentage change on all figures. It is evident that when scaled in this way, $\beta$ predominantly follows $u_{\infty}^{\prime} / U_{\infty}$, and $L_{u, \infty} / \delta+2$ is approximately constant. In $\S \S 4$ and 5 it was also shown that for cases with similar $L_{u, \infty} / \delta$, the mean profiles and spectrograms could look very different. Thus, while the integral scale likely does play some role, this role is small compared to the turbulence intensity and evolution history, at least for the test cases investigated herein.

\section{REFERENCES}

ADRIAN, R. J. \& YAO, C. S. 1986 Power spectra of fluid velocities measured by laser Doppler velocimetry. Exp. Fluids 5 (1), 17-28.

ANDERson, J. D. 2010 Fundamentals of Aerodynamics. McGraw-Hill Education. 
BAARs, W. J., Hutchins, N. \& MARUsic, I. 2016 Spectral stochastic estimation of high-Reynoldsnumber wall-bounded turbulence for a refined inner-outer interaction model. Phys. Rev. Fluids $1(5), 054406$.

BLAIR, M. F. $1983 a$ Influence of free-stream turbulence on turbulent boundary layer heat transfer and mean profile development, part ii-analysis of results. Trans. ASME: J. Heat Transfer 105 (1), 41-47.

BLAIR, M. F. $1983 b$ Influence of free-stream turbulence on turbulent boundary layer heat transfer and mean profile development, part i-experimental data. Trans. ASME: J. Heat Transfer 105 (1), 33-40.

Boyer, L. \& SEARby, G. 1986 Random sampling: distortion and reconstruction of velocity spectra from fast Fourier-transform analysis of the analog signal of a laser Doppler processor. J. Appl. Phys. 60 (8), 2699-2707.

CASTRO, I. P. 1984 Effects of free stream turbulence on low Reynolds number boundary layers. Trans. ASME: J. Fluids Engng 106 (3), 298-306.

Chauhan, K. A., Monkewitz, P. A. \& Nagib, H. M. 2009 Criteria for assessing experiments in zero pressure gradient boundary layers. Fluid Dyn. Res. 41 (2), 021404.

Coles, D. 1956 The law of the wake in the turbulent boundary layer. J. Fluid Mech. 1 (2), 191-226.

Comte-Bellot, G. \& Corrsin, S. 1966 The use of a contraction to improve the isotropy of grid-generated turbulence. J. Fluid Mech. 25 (4), 657-682.

Devinant, P., Laverne, T. \& Hureau, J. 2002 Experimental study of wind-turbine airfoil aerodynamics in high turbulence. J. Wind Engng Ind. Aerodyn. 90 (6), 689-707.

Dogan, E., HANSON, R. E. \& GANAPATHISUBRAmani, B. 2016 Interactions of large-scale free-stream turbulence with turbulent boundary layers. J. Fluid Mech. 802, 79-107.

Dogan, E., Hearst, R. J. \& Ganapathisubramani, B. 2017 Modelling high Reynolds number wall-turbulence interactions in laboratory experiments using large-scale free-stream turbulence. Phil. Trans. R. Soc. A 375 (2089), 20160091.

Dogan, E., Hearst, R. J., Hanson, R. E. \& Ganapathisubramani, B. 2019 Spatial characteristics of a zero-pressure-gradient turbulent boundary layer in the presence of free-stream turbulence. Phys. Rev. Fluids 4 (8), 084601.

Eitel-Amor, G., Örlü, R. \& Schlatter, P. 2014 Simulation and validation of a spatially evolving turbulent boundary layer up to $R e_{\theta}=8300$. Intl J. Heat Fluid Flow 47, 57-69.

Ertunç, Ö., Özyilmaz, N., Lienhart, H., Durst, F. \& Beronov, K. 2010 Homogeneity of turbulence generated by static-grid structures. J. Fluid Mech. 654, 473-500.

Esteban, L. B., Dogan, E., Rodríguez-López, E. \& Ganapathisubramani, B. 2017 Skin-friction measurements in a turbulent boundary layer under the influence of free-stream turbulence. Exp. Fluids 58 (9), 115.

Ferrante, A. \& Elghobashi, S. 2004 On the physical mechanisms of drag reduction in a spatially developing turbulent boundary layer laden with microbubbles. J. Fluid Mech. 503, 345-355.

GANAPATHISUBRAMANI, B. 2018 Law of the wall for small-scale streamwise turbulence intensity in high-Reynolds-number turbulent boundary layers. Phys. Rev. Fluids 3 (10), 104607.

HANCOCK, P. E. \& BRADShaW, P. 1983 The effect of free-stream turbulence on turbulent boundary layers. Trans. ASME: J. Fluids Engng 105 (3), 284-289.

HANCOCK, P. E. \& BRADSHAW, P. 1989 Turbulence structure of a boundary layer beneath a turbulent free stream. J. Fluid Mech. 205, 45-76.

Hearst, R. J., Buxton, O. R. H., Ganapathisubramani, B. \& Lavoie, P. 2012 Experimental estimation of fluctuating velocity and scalar gradients in turbulence. Exp. Fluids 53 (4), 925-942.

Hearst, R. J., Dogan, E. \& Ganapathisubramani, B. 2018 Robust features of a turbulent boundary layer subjected to high-intensity free-stream turbulence. J. Fluid Mech. 851, 416-435.

HeARST, R. J. \& LAVoie, P. 2015 The effect of active grid initial conditions on high Reynolds number turbulence. Exp. Fluids 56 (10), 185.

HUTCHINS, N. \& MARUSIC, I. 2007 Evidence of very long meandering features in the logarithmic region of turbulent boundary layers. J. Fluid Mech. 579, 1-28.

ISAZA, J. C., SALAZAR, R. \& WARHAFT, Z. 2014 On grid-generated turbulence in the near- and far field regions. J. Fluid Mech. 753, 402-426. 
Kang, H. S., Chester, S. \& Meneveau, C. 2003 Decaying turbulence in an active-grid-generated flow and comparisons with large-eddy simulation. J. Fluid Mech. 480, 129-160.

Kozul, M., Hearst, R. J., Monty, J. P., Ganapathisubramani, B. \& Chung, D. 2020 Response of the temporal turbulent boundary layer to decaying free-stream turbulence. J. Fluid Mech. 896, A11.

LARSSEN, J. V. \& DEVEnPort, W. J. 2011 On the generation of large-scale homogeneous turbulence. Exp. Fluids 50 (5), 1207-1223.

Laskari, A., De Kat, R., Hearst, R. J. \& Ganapathisubramani, B. 2018 Time evolution of uniform momentum zones in a turbulent boundary layer. J. Fluid Mech. 842, 554-590.

Lavoie, P., Djenidi, L. \& Antonia, R. A. 2007 Effects of initial conditions in decaying turbulence generated by passive grids. J. Fluid Mech. 585, 395-420.

MAKiTA, H. 1991 Realization of a large-scale turbulence field in a small wind tunnel. Fluid Dyn. Res. $8,53$.

Maldonado, V., Castillo, L., Thormann, A. \& Meneveau, C. 2015 The role of free stream turbulence with large integral scale on the aerodynamic performance of an experimental low Reynolds number S809 wind turbine blade. J. Wind Engng Ind. Aerodyn. 142, 246-257.

Marusic, I., Chauhan, K. A., Kulandaivelu, V. \& Hutchins, N. 2015 Evolution of zero-pressure-gradient boundary layers from different tripping conditions. J. Fluid Mech. 783, 379-411.

Marusic, I., McKeon, B. J., Monkewitz, P. A., Nagib, H. M., Smits, A. J. \& SREENIVASAN, K. R. 2010 Wall-bounded turbulent flows at high Reynolds numbers: recent advances and key issues. Phys. Fluids 22 (6), 065103.

Marusic, I., Monty, J. P., Hultmark, M. \& Smits, A. J. 2013 On the logarithmic region in wall turbulence. J. Fluid Mech. 716, R3.

Mohamed, M. S. \& Larue, J. C. 1990 The decay power law in grid-generated turbulence. J. Fluid Mech. 219, 195-214.

Monkewitz, P. A., Chauhan, K. A. \& Nagib, H. M. 2008 Comparison of mean flow similarity laws in zero pressure gradient turbulent boundary layers. Phys. Fluids 20 (10), 105102.

Perry, A. E., MARUSiC, I. \& Jones, M. B. 1998 New evolution equations for turbulent boundary layers in arbitrary pressure gradients. Sadhana 23 (5), 443-457.

PRANDTL, L. 1905 über Flüssigkeitsbewegung bei sehr kleiner Reibung. In Proceedings of the 3rd International Congress of Mathematicians, Heidelberg, 1904, Leipzig, pp. 485-491. B. G. Teubner.

Raushan, P. K., Singh, S. K. \& Debnath, K. 2018 Grid generated turbulence under the rigid boundary influence. J. Wind Engng Ind. Aerodyn. 182, 252-261.

Rodríguez-LóPez, E., BRUCE, P. J. K. \& BUXton, O. R. H. 2015 A robust post-processing method to determine skin friction in turbulent boundary layers from the velocity profile. Exp. Fluids $56(4), 68$.

Sharp, N. S., NeUSCAMman, S. \& WARHAFT, Z. 2009 Effects of large-scale free stream turbulence on a turbulent boundary layer. Phys. Fluids 21 (9), 095105.

Sillero, J. A., JimÉNEZ, J. \& Moser, R. D. 2013 One-point statistics for turbulent wall-bounded flows at Reynolds numbers up to $\delta^{+} \approx 2000$. Phys. Fluids 25 (10), 105102.

SMits, A. J. \& MARUSIC, I. 2013 Wall-bounded turbulence. Phys. Today 66 (9), 25.

Stefes, B. \& Fernholz, H. H. 2004 Skin friction and turbulence measurements in a boundary layer with zero-pressure-gradient under the influence of high intensity free-stream turbulence. Eur. $J$. Mech. B/Fluids 23 (2), 303-318.

Thole, K. A. \& Bogard, D. G. 1996 High freestream turbulence effects on turbulent boundary layers. Trans. ASME: J. Fluids Engng 118 (2), 276-284.

Vincenti, P., Klewicki, J., Morrill-Winter, C., White, C. M. \& Wosnik, M. 2013 Streamwise velocity statistics in turbulent boundary layers that spatially develop to high Reynolds number. Exp. Fluids 54 (12), 1629.

Wang, S., Zhou, Y., Alam, M. M. \& YANG, H. 2014 Turbulent intensity and Reynolds number effects on an airfoil at low Reynolds numbers. Phys. Fluids 26 (11), 115107.

WU, X. \& MoIN, P. 2009 Direct numerical simulation of turbulence in a nominally zero-pressure-gradient flat-plate boundary layer. J. Fluid Mech. 630, 5-41. 
Wu, X., Moin, P., Wallace, J. M., Skarda, J., Lozano-Durán, A. \& Hickey, J.-P. 2017 Transitional-turbulent spots and turbulent-turbulent spots in boundary layers. Proc. Natl Acad. Sci. 114 (27), E5292-E5299.

Wu, X., Wallace, J. M. \& Hickey, J.-P. 2019 Boundary layer turbulence and freestream turbulence interface, turbulent spot and freestream turbulence interface, laminar boundary layer and freestream turbulence interface. Phys. Fluids 31 (4), 045104.

YoU, J. \& ZAKI, T. A. 2019 Conditional statistics and flow structures in turbulent boundary layers buffeted by free-stream disturbances. J. Fluid Mech. 866, 526-566. 